\title{
Hand synergies: Integration of robotics and neuroscience for understanding the control of biological and artificial hands.
}

\author{
Marco Santello, ${ }^{1, \star}$ Matteo Bianchi, ${ }^{2,3}$ Marco Gabiccini, ${ }^{2,3,4}$ \\ Emiliano Ricciardi, ${ }^{5}$ Gionata Salvietti, ${ }^{6}$ Domenico Prattichizzo, ${ }^{6,3}$ \\ Marc Ernst, ${ }^{7}$ Alessandro Moscatelli, ${ }^{8}$ Henrik Jorntell, ${ }^{9}$ \\ Astrid M. L. Kappers, ${ }^{10}$ Kostas Kyriakopoulos, ${ }^{11}$ Alin Abu Schaeffer, ${ }^{12}$ \\ Claudio Castellini, ${ }^{12}$ Antonio Bicchi ${ }^{2,3, \star}$ \\ ${ }^{I}$ School of Biological and Health Systems Engineering, Arizona State University, \\ Tempe, AZ, U.S.A. \\ ${ }^{2}$ Research Center 'E. Piaggio', University of Pisa, Pisa, Italy \\ ${ }^{3}$ Advanced Robotics Department, Istituto Italiano di Tecnologia (IIT), Genova, Italy. \\ ${ }^{4}$ Department of Civil and Industrial Engineering, University of Pisa, Pisa, Italy \\ ${ }^{5}$ Clinical Psychology Branch, Pisa University Hospital, Pisa, Italy \\ ${ }^{6}$ Department of Information Engineering and Mathematics, University of Siena, Siena, Italy \\ ${ }^{7}$ Department of Cognitive Neuroscience and CITEC, Bielefeld University, Bielefeld, Germany \\ ${ }^{8}$ Department of Systems Medicine and Centre of Space Bio-Medicine, Università di Roma "Tor Vergata", \\ 00173, Rome, Italy \\ ${ }^{9}$ Neural Basis of Sensorimotor Control, Department of Experimental Medical Science, Lund University, \\ Lund, Sweden \\ ${ }^{10}$ Human Movement Sciences, Vrije Universiteit, Amsterdam, The Netherlands \\ ${ }^{11}$ School of Mechanical Engineering, National Technical University of Athens, Greece \\ ${ }^{12}$ DLR-German Aerospace Center, Institute of Robotics and Mechatronics, Oberpfaffenhofen, Germany
}

Keywords: movement; force; biomechanics; electromyography; motor control.

${ }^{\star}$ Corresponding authors

Marco Santello

School of Biological and Health Systems Engineering

501 East Tyler Mall, ECG Building, Suite 334

Arizona State University

Tempe, AZ 85287-9709, U.S.A.

Ph.: +1-480-965-8279

Fax: +1-480-727-7624

E-mail: marco.santello@asu.edu
Antonio Bicchi

Research Center 'E. Piaggio'

University of Pisa

Largo Lucio Lazzarino 1

56122 Pisa, Italy

Ph: +39-050-2217050

Fax +39.050.2217051

E-mail: antonio.bicchi@ unipi.it 


\section{Abstract}

The term 'synergy' - from the Greek synergia - means 'working together'. The concept of multiple elements working together towards a common goal has been extensively used in neuroscience to develop theoretical frameworks, experimental approaches, and analytical techniques to understand neural control of movement, and for applications for neurorehabilitation. In the past decade, roboticists have successfully applied the framework of synergies to create novel design and control concepts for artificial hands, i.e., robotic hands and prostheses. At the same time, robotic research on the sensorimotor integration underlying the control and sensing of artificial hands has inspired new research approaches in neuroscience, and has provided useful instruments for novel experiments.

The ambitious goal of integrating expertise and research approaches in robotics and neuroscience to study the properties and applications of the concept of synergies is generating a number of multidisciplinary cooperative projects, among which the recently finished 4-year European project "The Hand Embodied" (THE). This paper reviews the main insights provided by this framework. Specifically, we provide an overview of neuroscientific bases of hand synergies and introduce how robotics has leveraged the insights from neuroscience for innovative design in hardware and controllers for biomedical engineering applications, including myoelectric hand prostheses, devices for haptics research, and wearable sensing of human hand kinematics. The review also emphasizes how this multidisciplinary collaboration has generated new ways to conceptualize a synergy-based approach for robotics, and provides guidelines and principles for analyzing human behavior and synthesizing artificial robotic systems based on a theory of synergies. 


\section{Introduction}

The human hand is an extraordinarily sophisticated and versatile sensorimotor system. Controlling the large number of elements of the hand, such as muscles, bones, and joints, as well as integrating multiple sensory modalities, are complex tasks the Central Nervous System (CNS) must deal with.

As infants, we use our hands as a sensory organ to learn the properties of the world around us, e.g., whether a surface is cold or hot, rough or smooth, hard or soft. As early motor developmental milestones are conquered and well before we learn how to walk or to verbally communicate, we acquire the ability to perform well-directed reaching movements towards objects that capture our attention, pre-shape our hands to ensure that we can grasp objects as we complete our reach, and oppose the thumb to the other fingers to firmly grasp and manipulate objects. As the CNS continues to mature, we become more skilled at using our hands as motor organs, through which we gradually learn to perform a wider gamut of manual actions. Some of these actions may require the ability of moving individual digits independently when, for example, we need to pick and manipulate a small object, or learn to play a musical instrument. Other actions may require the use of all digits in a synergistic fashion, i.e., by conjointly closing or opening all digits in a coordinated way [1].

Given the critical role that the hand plays in activities of daily living, hence quality of life, as well as to better understand how to improve functional recovery of hand control after neurological or traumatic injuries, many studies have been devoted to characterize the way the CNS controls the hand. As hand function emerges from the interplay among a large number of sensory and motor elements, neural control of the hand has been studied across many levels of the CNS and through a wide variety of experimental approaches. The quantification of the spatial 
and temporal coordination of multiple hand muscles, joints, and digit forces has led a number of investigators to develop and test the theoretical framework of hand synergies.

\section{Synergies}

In the large body of studies on neural control of movement (for reviews the reader is referred to [2-7]) "synergies" have been defined in several different ways depending on the level(s) and scale of the sensorimotor system being investigated, including but not limited to motor units, muscles, and joints (for details see [8]). Thus, at the level of motor units, common neural input in the time and/or frequency domain (synchrony and coherence, respectively) could be viewed as a form of synergistic control as it constrains the timing at which multiple motor units are activated (for review see [9]). At the muscle level, muscle synergies have been defined as patterns of muscle activity whose timing and/or amplitude modulation enable the generation of different movements (for review see [10]). When examining motor output rather than neural drive underlying movement production, synergies have also been identified and defined as covariation patterns that constrain in a systematic way angular excursions at multiple joints, e.g., hand postural synergies ([11-13]; for review see [8]), or covariation patterns among digit forces (for review see [14]).

Regardless of the level of the CNS at which synergies are defined, the main implication that is shared across the different definitions of synergies is that multiple degrees of freedom are controlled within a lower dimensional space than the available number of dimensions. It has been proposed that in the intact nervous system, synergies allow to flexibly adapt to different task conditions, e.g., the amplitude and/or temporal relations among several muscles can be changed to generate different motor behaviors, or the same behavior across different task conditions. However, injury to the nervous system would interfere with the ability of the sensorimotor 
system to flexibly combine synergies, thus leading to abnormal synergies $([15,16]$; for a review see [6]).

As the number of studies of hand synergies in animal and human models grew and refined analytical and experimental approaches, over the past decade roboticists have started to investigate the potential applications of hand synergies for artificial hands $([17,18])$. This work has led to the successful application of the concept of synergies [17] and the creation of novel design and control concepts for robotic hands and prostheses, while providing experimental tools and new research approaches for neuroscience.

The aims of this review are to provide an overview of the neuroscientific bases of hand synergies - meant as common patterns of actuation of the human hand - and to report how robotics has leveraged the insights from neuroscience for innovative design in hardware and controllers for biomedical engineering applications, including myoelectric hand prostheses, devices for haptics research and human-machine interactions, and wearable sensing of human hand kinematics. We also describe insights that robotics research on artificial hand design and control has provided to neuroscience research. This review mainly focuses on the most significant achievements of the international cooperation project called "THE Hand Embodied"1 and is organized in four sections. The first section describes the synergistic organization underlying motor control of the human hand. We will review how such an organization can be observed at different levels - postural, muscular, and neural - and quantified through different techniques - motion tracking, electromyography (EMG), and brain functional imaging. The second section describes how the concept of synergy has been exploited in robotics to develop tools for the analysis, modeling, and synthesis of artificial robotic hands, with potential

\footnotetext{
1 "THE Hand Embodied" project was supported by the European Community under CP grant no. 248587, within the FP7-ICT-2009-4-2-1 program "Cognitive Systems and Robotics" (01.03.2010 - 28.02.2014).
} 
applications for human-robot interaction and prosthetics. The third section focuses on the relation between sensing and human hand synergies, and in particular on how the concept of dimensionality reduction might apply to the sensory domain of hand control, in the framework we define as sensory synergies. Furthermore, we consider how sensory and motor elements can be combined together into the paradigm of sensory-motor synergies. Analogies and applications in robotics are also discussed. The fourth and last section introduces open questions and directions for future research built on leveraging the complementary questions and approaches used in robotics and neuroscience to advance our understanding of neural control of the hand, as well as biomedical and robotic applications.

\section{Hand synergies: Motor control}

The taxonomy proposed in [19] divided the large number of hand postures into two main categories: power and precision grips. The main insight of this taxonomy was the definition of hand postures based on a functional gradient. Thus, tasks that demand large forces are performed using hand postures that are characterized by large contact areas, often involving the palm of the hand and several digits. In contrast, precision grips would be used to perform tasks that require small but finely controlled forces, often exerted through the fingertips. However, the examination of digit joint kinematics extracted from static hand posture revealed a different picture than the aforementioned taxonomy. Specifically, hand postures used to grasp a wide variety of imagined objects could be described by a very small number of linear combinations of joint angles, i.e., principal components or postural synergies (or eigenpostures) [11]. Furthermore, it is also possible to observe a gradient in hand eigenpostures, where lower-order principal components reflect covariation patterns for metacarpal-phalangeal (MCP) and 
interphalangeal (IP) joints - which are mainly responsible for coarse hand opening and closingand higher-order principal components corresponding to finer hand shape adjustments. These observations, which have been replicated with real objects [12] and in many other experimental settings (see [8] for a review) revealed that the apparent complexity associated with having to control multiple digit joints might be addressed by constraining them to move in a synergistic fashion. In other words, the problem of controlling many joints is not as complex as it seems, especially when not all joints can or should be moved independently from other joints.

As reviewed in [8], the synergistic organization in the motor domain can be defined according to the level at which the analysis is performed. In the following sections, we will review the main concepts related to how synergies have been defined at kinematic or postural level, at the muscular level, and at the neural level.

\subsection{Kinematic redundancy and biomechanical constraints}

The biomechanical architecture of the hand provides some insights into how the CNS might implement a synergistic control of digit motion and forces (for a review see [20]). Briefly, the tendons of extrinsic finger flexors and extensors cross several joints. Therefore, tension exerted on a tendon would generate torques at several joints. Furthermore, passive linkages exist among hand muscles, and in non-human primates these connections can transmit tension across tendons [21]. Both of these observations indicate that motion at multiple joints and/or digits can occur without the need for, or the existence of, neural drive to multiple hand muscles. However,

and as discussed in [6], the CNS can override - to some extent - these tendencies for 'unwanted' widespread motion at multiple fingers, as it happens following training in performing individuated finger movement necessary for typing or piano playing. 


\subsection{Muscle synergies}

As mentioned above, synergies can be also studied at the muscular level. The leading hypothesis of the so-called "muscle synergies" is that multiple muscles can be activated as a unit by varying their timing and/or neural drive (quantified as EMG). The combinations of these multi-muscle activation patterns would therefore underlie the control of given movements, e.g., postural control, gait, or arm movements, across different task conditions or requirements $[4,10,22]$.

With respect to hand control, and in addition to the above-described peripheral constraints on independent digit motion and contact forces, it has been proposed that the organization of neural inputs to motor nuclei of hand muscles in the spinal cord might also contribute to their synergistic activation. Specifically, common neural inputs to motor units of hand muscles have been described for extrinsic and intrinsic hand muscles during grasping [23,24]. Interestingly, common neural input to hand muscle motor unit pairs appear to be distributed along a functional gradient, where muscles involved in synergistic finger control tend to receive stronger common neural input than muscles involved in fine modulation of digit movement and forces (extrinsic and intrinsic hand muscle, respectively) ([25,26]; for a review see [9]). Studies of hand muscle synergies quantified at the motor unit population level through interference EMG have revealed that the activation of multiple muscles scales with total grip force during three-digit grasping [27], thus complementing the above observations of correlated neural input to motor unit pairs. Non-human primate studies have further quantified correlations in EMG amplitude of hand muscles recruited for grasping objects with different shapes and sizes [28,29]. Synergistic control of multiple hand muscles has also been described by studies of whole-hand grasping in humans [30] and finger spelling [30,31]. It should be noted that the 
analysis of hand synergies at the muscular or kinematic level are likely to reveal phenomena that lie on a functional continuum, i.e., dynamic and static hand postural synergies may be related to, or mediated by, hand muscle synergies. This view is supported by a recent study showing a close correspondence between muscle synergies and kinematic synergies in a reach-grasp-pull task [32].

An important question is whether hand synergies are learned or inborn. The earliest form of hand synergy is probably the 'palmar grasp reflex' that is found at birth, and through which the whole hand closes in response to a stimulation of the palm of the hand. Movement of individual fingers and thumb follow at 10-12 months of age, thus laying the foundations for more complex hand movement patterns that will gradually be mastered over the years [33]. It is tempting to speculate that this gradual evolution from somewhat basic hand movement patterns (e.g., whole hand opening and closing) to movements characterized by greater motion independence among the fingers might correspond with the above-described eigenpostures. Experimental observations point out to the high degree of similarity in eigenpostures among individuals, both in laboratory tasks (e.g., [11]) and activities of daily living [34], as well as across manual tasks [35]. At the same time, the role of training and intensive practice can, to some extent, modify these stereotypical finger movement patterns [36]. However, the degree of plasticity with which motor commands can adapt to override synergistic finger movement patterns is not fully understood. A better understanding of the extent to which hand synergies can emerge through sensorimotor experience and interactions with the environment will be extremely valuable scientifically and in terms of bio-inspired technological applications (see Section 2). 
In summary, the organization of neural inputs to hand muscles limits the extent to which the CNS can independently activate individual hand muscles, thus control individual digit forces and movement. Such limitation is captured by the tendency of neural activity measured across motor unit pairs and populations to be modulated in a synergistic fashion across multiple muscles. The next section examines constraints and neural correlates of hand synergies at higher levels of the CNS.

\subsection{Neural bases of hand synergies}

As reported in the previous sections, the control of the hand can be described by consistent spatial-temporal coordination patterns in the kinematic, kinetic, and muscle domains across a wide variety of tasks. Thus, the critical question of whether and to what extent hand synergies are represented in the CNS arises. In other words, are synergies the best models to describe how the sensorimotor system deals with redundant solutions to the control of multiple degrees of freedom [2,37-39] or, rather, are synergies the 'real alphabet' that the motor-related brain areas rely on to compose the 'words' of different hand movements [40-42]?

The compelling hypothesis is that synergistic control of the hand would not simply represent an effective theoretical approach to explain how the CNS solves the redundancy problem, but could also characterize the way movements and actions are represented and recognized at the neural level. Brain regions involved in hand action representation and motor planning are distributed in a well-identified fronto-parietal cortical network, the so-called 'grasp circuit' [43]. Within these action-sensitive regions, the neural representations of different features of movements (e.g. target representation, final goal, hand-object interaction, etc.) appear to be extensively distributed but topographically organized [44-46]. Nonetheless, the nature of the specific neural information encoded within these regions is still ill-defined. Of particular 
relevance to this review article is the question about the structural and functional organization of hand movement control within brain regions such as primary motor cortex, supplementary motor area and premotor cortex, from which the cortico-spinal transmissions originate to control motor units.

Historically, the concept of motor somatotopy was introduced based on work on humans and animal models. This concept proposes that primary motor cortex is topographically arranged into sub-segments that control a single effector, or a subset of effectors, e.g., muscles or joints [47-50]. Whereas a coarse arrangement of body limbs (e.g. hand, mouth or face areas) has been confirmed within primary motor areas, the intrinsic organization of the distinct limb-specific clusters has often been challenged ([51-53]; see also review by [20]). For instance, in the handrelated areas of primary motor cortex, single finger representations appear to be distributed along overlapping patches that lack an elementary functional organization, and are even interconnected, thus favoring a hand-movement coding based on multi-joints and multi-muscles modules [21,54-56]. In addition, neurophysiological studies have indicated that single neurons within primary motor and premotor areas exhibit a heterogeneous organization that includes lowlevel representations of single digit control - as predicted by a somatotopic functional organization $[47,51]-$, to the higher-level description of full behaviorally-relevant motor acts, which would favor an action goal-oriented organization [57-59].

The above-described behavioral and physiological observations indicating that hand postures can be effectively characterized as linear combinations of a small number of synergies offer an alternative and more comprehensive theoretical description of motor functional organization. While favoring the idea of a modular definition of motor acts, a synergy would represent a way for the CNS to (1) simplify motor control through a dimensionality reduction 
strategy, (2) produce complex movements through a wide number of weighted combinations of neuronal populations, and (3) spatially and temporally coordinate multiple elements of individual digits through a task-specific interaction with the biomechanical constraints of the effector. An invariant representational structure for simple and complex hand movements, independent from the somatotopic arrangement of the digits, has been recently demonstrated in sensorimotor cortex, thus supposing the existence of an 'organizing mechanism' that models the similarity between movement-related response patterns across individuals [60].

So far, however, limited evidence exists of synergy-based neural representations of hand synergies in motor cortical areas. Specifically, direct intracortical microstimulation in nonhuman primates [7,41] and indirect transcranial magnetic stimulation in humans [40,61] over primary motor cortex can elicit synergistic finger movement patterns. Nonetheless, until recently no study has directly associated neural responses within primary motor areas in humans to synergy-based hand control, or dimensionality-reduction strategies in motor planning at the cortical level.

To better understand the brain functional architecture that mediates hand synergies, a recent study [62,63] combined machine learning and multivariate approaches methods to neuroimaging $[64,65]$ and integrated these data with motor control models, as defined via behavioral or electrophysiological measurements. Specifically, multivariate techniques allowed to directly assess whether brain activity at a cortical level encodes hand movements through the "language" of postural hand synergies. A multimodal experimental paradigm integrated patterns of neural responses during grasping movements as measured with functional magnetic resonance imaging (fMRI) with kinematic and EMG-based models of hand synergies. Preliminary results revealed a significantly higher accuracy of the synergy-based model than a somatotopy-based 
model of functional organization in predicting movement-specific patterns of neural response. Importantly, the synergy-based model also indicated that synergies are encoded in primary motor areas associated with hand control in a consistent fashion across subjects. If confirmed, these observations of a 'high-level' encoding of individual hand postures through synergies would provide, for the first time, evidence for neural correlates of functional sensory-motor modules in the brain. In addition, the specificity of the patterns of neural response elicited during different movements was exploited to predict hand postures directly from fMRI activity. This provides novel ways of decoding task-specific patterns of neural responses from motor control brain regions, as recently attempted using electroencephalography [66] and envisioned for robotic applications, such as more effective methods to acquire, localize, and decode signals for brainmachine interfaces and prosthetic applications.

From both theoretical and methodological perspectives, motor synergies account for an extensive, but still relative, amount of neural content of information, as quantified by measures of variance of brain activity [62]. This suggests that primary motor areas also process other action-related features, such as force production [67], final posture [57,68,69], or even single digit movements [70] and joint angles [71]. Therefore, further studies should also address the relative contribution of peripheral constraints versus central commands in generating coupled motion of the digits.

\section{From biology to robotics}

Attracted by the above-described experimental observations of synergistic patterns of finger motion, in the last decade roboticists have proposed a geometric model of hand synergies, which is applicable to the pre-grasping phase, i.e., before actual contact with the grasped object. 
The underlying concept for this model - and for a general geometrical interpretation of the concept of synergies - is dimensionality reduction. This dimensionality reduction refers to the reduction of the number of degrees of freedom (DOFs) of the human hand that can be controlled in an independent manner [72], as well as of the space of possible (most frequent) hand postures that can be actively controlled. One of the first software applications of this concept was provided by [18] as a means to reduce the number of dimensions of the large search space of robotic grasp planning with dexterous hands. The reduced computational burden allowed by a low-dimensional hand posture subspace allowed on-line grasp synthesis at a rate compatible with effective user interaction [73], and the reduction of the control variables [74]. At the same time, the geometric model of hand synergies suggested novel approaches for the design and control of robotic hands with a reduced number of aggregated DOFs. The first notable mechanical implementation of postural synergies was made in [17], where a two-eigenposture mechanism design combined and drove a 17-DOFs 5-fingered robot hand through a train of pulleys with different radii.

Although the adoption of the concept of synergies for the development of robotic hands brought a significant simplification in terms of design and control complexity, it opened novel research questions: (1) Is anthropomorphism the best choice to implement and exploit the synergistic model in robotics? and (2) Is it possible to define a general framework to extend and map human hand synergies to robotic hands with dissimilar structure from a human hand? Question (1) is related to the debate on the usefulness of anthropomorphism in robot motion and design. Considerations on safety in human-robot physical interactions [75] and social interactions [76-78] motivate the anthropomorphic approach. For example, in scenarios where humans and robots have to cooperate to execute specific tasks, anthropomorphic robot motion 
can be more easily predicted by humans who could therefore better adapt to robot actions and avoid possible injuries.

The suggestion to leverage the concept of human hand synergies for biologically-inspired designs of robotic hands can also be extended to other structures, such as upper and lower limbs. For example, in [79], two suitable non-linear kinematic synergies were constructed for the lower part of the body of a humanoid robot and exploited to increase its capability to balance dynamically against unforeseen disturbances, e.g. from external forces or due to manipulation of unknown loads.

In these cases, too, anthropomorphic robot motion inspired by human example could leverage the concept of synergies to enable more effective human-robot interactions (HRI) and co-adaptation, as well as artificial control. Furthermore, the spatial and temporal coordination of arm, wrist, and hand movements during reach-to-grasp points to a control strategy that constrains the action of multiple effectors and joints to achieve a high-level goal, i.e., transporting the hand in a way that optimizes the likelihood of successful object grasp and manipulation [80-85]. In this context, it should be noted that hand kinematic synergies have been quantified also during reach-to-grasp, indicating that the temporal evolution of hand shape is spatially and temporally coordinated with the control of shoulder and elbow kinematics responsible for hand transport [13].

The above-described biologically-inspired motor control strategies have been used in robotics also to define closed-loop grasp planning schemes to ensure anthropomorphism of robot motion, convergence to desired task goals, and human-like adaptive robot behavior. To achieve these goals, research has focused on mapping human motion to anthropomorphic robot motion through non-linear, constrained optimization methods [86-89]. The anthropomorphic robot 
trajectories are then projected into low-dimensional manifolds of upper limb "principal components", where appropriate Navigation Function models can be trained [90].

As specified in the above research question (2), the problem of mapping human to robot motion is particularly relevant also for the correct definition of a general framework for controlling robotic hands independently from their kinematics and mechanical design. This approach entails designing control algorithms for an anthropomorphic hand model with predefined synergies and mapping the resulting finger motions onto several robotic hands. This procedure can be implemented at different levels, e.g. at the joint level [73], in the Cartesian space [91], and considering the relation among hand poses [92]. However, the success of these approaches strictly depends on the kinematic structure of the robotic device, and thus cannot be easily generalized. To tackle this problem, a different solution was proposed in [93], where the authors introduced the concept of virtual objects and a mapping from humans to robots designed in the manipulated object domain. The role of virtual object can be played, for example, by a virtual sphere, which can be defined as the minimum volume sphere that includes a set of reference points on the fingertip of the human hand. Thus, motion of the human hand generates motion of the reference points, and consequently of the virtual sphere. Another example of this approach can be found in [94], where a rigid motion of the object center and a non-rigid strain (e.g., variation of the sphere radius) were used.

At the same time, by defining in an analogous manner a virtual sphere also on the robotic hand, it is possible to implement such object-based mapping procedure by imposing that the rigid and non-rigid motions captured by the virtual object in the human hand are replicated by the object defined on the robotic hand. Other more general virtual object geometries were defined in $[95,96]$. The validity of the whole object-based mapping procedure was demonstrated with 
different robotic hand models, considering both motions and exerted forces, in bilateral teleoperation tasks and through software simulation [97,98] (Fig. 1).

\section{[INSERT FIGURE 1 APPROXIMATELY HERE]}

These mapping approaches can lead to standardization among control strategies of robotic hands with dissimilar structures, thus enabling to fully exploit a synergistic organization in a device-independent manner. This theoretical framework is reminiscent of the concept of motor equivalence, originally introduced by [100], which is a topic of ongoing investigation (e.g., [101]). This also allows to replicate an organized set of synergies in the artificial hand, ordered by increasing complexity, so that a correspondence can be attained between any specified task set - in terms of a number of different grasps and explorative actions - and the least number of synergies whose aggregation makes the task feasible.

\subsection{Soft synergies for soft hands}

In the previous section, we showed how the geometric model of postural synergies can be successfully exploited to implement synergies for software and hardware design. This approach enables to adopt a model of the hand with a number of independent actuators that is smaller than the number of joints. However, this approach does not necessarily imply that the hand will move and comply with the shape of the grasped object. To extend the applicability of this synergy model to the correct implementation of force generation and distribution in the robotic hand grasp and contact force control, the soft-synergy model was introduced to factor in the mechanical compliance of the hand musculo-tendinous system [102]. 
In this model, the physical hand is attracted by an elastic field towards a reference hand identified by the geometric 'synergy coordinates' (as defined in [11] and discussed in the previous section). At the same time, the forces arising from contact with objects in the environment and mediated by the impedance of the hand-object mechanical system repels the physical hand from the reference hand, thus being responsible for force distribution during grasp. Numerical results obtained by this model indicate that the same dominant synergies (i.e., principal components) observed from human pre-grasp postural data are also crucial for establishing force distributions to perform stable grasps [102,103] .

Such soft-synergy paradigm has triggered renewed interest for robotic grasp analysis and synthesis problems, together with the need of developing novel analytical tools to deal with the synergistic compliant coupling of a subset of DOFs. For example, the soft-synergy paradigm has enabled the definition of a rigorous procedure to establish the number of synergies that guarantees grasp stability and efficiency, depending on the task to be performed and the type of considered embodiment. To this aim, [104] proposed a quasi-static model and defined grasp structural properties related to contact force and object motion controllability. Here, the compliant model of soft synergies was assumed and different sources of compliance in the manipulation system were considered. Further studies focused on extending the manipulability analysis to synergy-actuated hands by employing the concept of manipulability ellipsoids and introducing new manipulability indexes [105]. This approach allowed the identification of the directions in the input and output spaces that maximize this manipulation efficiency metric. These directions could also be used as a tool to assess the manipulation performance of robotic hands by taking directly into account underactuation (i.e., the use of less degrees of actuation than DOFs) and compliance. A general grasp analysis framework for underactuated hands with 
compliance, which considers also pre-loading conditions, was then presented by [106]. Here, new definitions of structural properties of general manipulation systems and novel matrix factorization procedures were introduced to define a general approach to control the Cartesian grasp compliance in synergistically underactuated hands with variable impedance joints [107].

However, although the idea of soft synergy actuation represents an elegant solution to the problem of simple hand design, combining natural motion from the postural synergy approach with adaptability through compliance, its mechanical implementation is technically challenging. To address this issue, a soft-synergy model was translated into the corresponding adaptive synergy model, which can be identified through transmission matrix and joint stiffness. Based on the above considerations, the Pisa/Italian Institute of Technology (IIT) SoftHand [108,109], a joint venture between the University of Pisa and IIT, represents a promising approach to the design of soft-synergy inspired hands through adaptive underactuated mechanisms (Fig. 2).

\section{[INSERT FIGURE 2 APPROXIMATELY HERE]}

The Pisa/IIT SoftHand is an anthropomorphic robotic hand with 19 joints but only one actuator, which implements the soft-synergy model along the first postural synergistic direction through its corresponding adaptive synergy counterpart. This artificial hand exploits an innovative soft design of joints and ligaments, which enables a high level of compliance and adaptation to objects of different shape and dimensions. The mechanical design is parameterized by a transmission matrix and a joint stiffness matrix that allow the hand to exhibit the same behavior as the soft synergy model of human hand around a local equilibrium configuration. At the same time, the Pisa/IIT SoftHand design ensures high robustness, which allows the hand to exploit the external physical constraints to increase manipulation performance. Thanks to its 
simplicity, compliance, and robustness, the Pisa/IIT SoftHand represents an ideal platform for the development of a novel prosthesis $[110,111]$, thereby opening interesting perspectives in prosthetics design and robotic rehabilitation. At the same time, EMG synergy-based approaches can be profitably exploited to increase control performance in HRI and prosthetics, as analyzed in the next section.

\subsection{EMG-Based Human-Robot Interaction Applications and Prosthetics}

In the framework of rehabilitation and assistive robotics, surface electromyography (sEMG) has been used as a human-machine interface for decades. Typically, a given number of sEMG signals are processed in real-time to control a rehabilitation or assistive device, e.g., an exoskeleton or a hand prosthesis, and enforcing at least a rough form of open-loop control by the prosthetic user. This is called myoelectric control, as it potentially enables control of the mechatronic device by using muscular activity in a way that mimics how the subject used to control his musculature before the loss of a limb. For the standard one-DOF hand prosthesis case, it is customary to have two sEMG sensors located on the residual wrist flexor and extensor muscles to control the closing and opening of the prosthesis.

As opposed to this traditional approach, which has been and remains the clinical standard since the 60 's, controlling recent research prototypes of prosthetic hands relies on a larger number of sEMG electrodes, typically up to several tens of them, to better capture the electrical activity or the residual musculature and therefore decode the intent of the user [112]. Since such a well-tuned set of sEMG signals reflects quite faithfully the underlying residual muscle activity [113], the question arises as to whether the concept of muscle synergies can be of practical use for prosthetic applications. If so, can synergistic muscle activations be detected in the highdimensional sEMG signal space? Furthermore, does this signal space measured in the residual 
musculature of individuals with upper limb loss reflect the muscle synergies as found in the intact CNS? Lastly, can these muscle synergies be used to effectively simplify the task of the myoelectric controller, e.g., to 'translate' the motor plan or intent of the user into specific hand postures and/or torques/forces?

These issues are controversial. In [114], the authors reported that multi-muscle activation patterns can be detected across sEMG signals. This is hardly surprising as sEMG electrodes directly detect the neural activity of the motoneurons, although in a mixed form [115]. In the works cited above, Principal Component Analysis revealed that four linear combinations of sEMG signals could account for up to $90 \%$ of the sEMG signal variance, thus suggesting the existence of muscle synergies which are reminiscent of the above-described EMG-based synergies described for grasping and finger spelling in humans and non-human primates, as discussed in section 1.2

It remains to be defined whether a synergy-based approach for decoding multiple sEMG signals has practical applications for myoelectric hand prostheses controllers. First, to evaluate such synergy-based approach, a fairly large number of sEMG signals with good signal-to-noise ratio is required, and this might be challenging in individuals with upper limb loss. Additionally, sEMG signals from amputees are known to change with time, e.g., as it happens with practice with self-powered prostheses [116]. Second, extracting sEMG synergies to be fed to a machinelearning algorithm as an alternative to using the original, fully-dimensional input space is tantamount to compressing the input signal with potential loss of information, hence accuracy. However, the results of two recent methodological approaches appear to mitigate the above concerns. First, we will introduce the concept of incremental learning used to map multi-sEMG signals to multi-DOF devices: for example, in [117], an eight-dimensional sEMG space is used 
to finely control one of the most advanced commercially-available hand prostheses, the $i$-LIMB (Touch Bionics Ltd.) in a teleoperated scenario using an incremental machine learning method. Then we will describe how sEMG signals from two muscles with opposite mechanical actions can be used to map the user's impedance and position control in a synergistic fashion onto an advanced robotic hand designed according to the concept of soft-adaptive synergies [118]. Whether the theoretical framework of sEMG-based synergies is of relevance here remains an open question, but these recent studies, as well as other investigations of using multi-sEMG signals for robotic hand control (see e.g. [119] or the survey in [120]), seem to pave the way towards a successful exploitation of synergistic concept in prosthesis control.

Mapping EMG from multiple muscles to multi-DOFs devices.

Whereas multi-fingered hand prostheses, prosthetic elbows, wrists and shoulders are now available for use in the rehabilitation clinics, in general control of these devices by the amputee is still largely an open issue. The standard approach is to use sEMG as the input signal to a machine learning- (ML-)based system; there is evidence in the scientific literature (e.g., [121123]) that, even decades after the operation, a surprising wealth of information can still be extracted from residual muscles. In other words, an appropriate set of sEMG sensors, coupled with a smart ML system, can effectively translate surface signals into control commands enacted upon the subject's will.

When considering sEMG from multiple residual muscles, interfacing these signals with a multi-DOF device implies creating a direct mapping between a multi-EMG pattern associated with a given movement, and the corresponding prosthetic control command. If the original multimuscle EMG pattern associated with movement can be exploited (that is, the EMG pattern that the subject would have recruited before the amputation), natural control can be enforced; that is, 
there is the possibility of letting the amputee controls the prosthesis in the most transparent way. On the other hand, as the amputee learns how to use a prosthesis, if the prosthesis reacts reasonably well, chances are that new multi-muscle patterns, or synergies meant as a reorganization of the original sEMG pattern, will be developed in order to obtain an optimal MLbased control.

At the time of writing, the major factor hindering an effective deployment of ML-based control systems in the clinics is the issue of reliability [124,125]. ML-based control has the potentiality of actuating many DOFs simultaneously and proportionally, that is to dramatically increase the acceptance of self-powered prostheses. However, as it happens with all statistical methods, it cannot assure that a certain pattern will be stably recognized. Indeed, ML-based methods relying on classification are discrete decision systems (on-off) that suffer from the inherent ambiguity of the signal as it crosses the boundary between two decision zones of the input space. Moreover, surface signals are influenced by a number of factors such as sweat, sensor positioning and muscle fatigue.

The concept of simultaneous and proportional (s/p) myocontrol defined in [115] can be regarded as an attempt to improve the reliability of ML-based myocontrol. S/p control directly maps the detected multi-muscle EMG onto patterns to simultaneous activation of several DOFs thanks to a regression method, rather than a classifier. Although still debated, the superiority of regression with respect to classification lies in (a) the capability of giving graded control as opposed to on-off, and (b) the fact that small errors in regression would likely not lead to a catastrophic result, but rather to slight instabilities in force application by the prosthesis. For these reasons, there is a definite trend in the community to employ s/p control in place of classification $[86,120,126]$. 
It should be noted that the vast majority of ML methods employ a one-shot initial calibration phase. To make this calibration sufficiently robust, the usage of an incremental machine learning approach was recently proposed. Incrementality means that the function approximation can be further refined after the initial calibration phase has finished; in fact, rather than calibration, the term model update is employed in this case [117,127]. As instabilities in the control can be therefore corrected as they happen, this approach has been called interactive learning.

\section{Minimalistic EMG Mapping: Tele-impedance approach}

In the previous paragraph, we have described several approaches used for mapping multiple sEMG signals to control prosthetic hands. In this paragraph, we propose a different approach: using the minimal number of EMG signals to control a synergy-inspired robotic hand, i.e. the Pisa/IIT SoftHand described in section 2.1. Furthermore, below we discuss a novel control concept beyond the classic position control approach, i.e., tele-impedance.

When humans interact with the external environment, they are able to produce restoring forces with respect to environmental displacement [128] by modulating limb impedance through muscle co-contraction. It has been proposed that tele-operation tasks, such as operating a prosthetic hand through myoelectric control or remote control of a robotic gripper, can be performed using compliant (soft) slave robots and time-to-time modulation of their impedance throughout the task. [129] proposed an approach to overcome stability problems in forcereflecting tele-operation and enable a more human-like task execution [130]. In this approach, user intent is incorporated into the control command, which includes both the desired motion or equilibrium position and stiffness profile estimated on the master side through a suitable humanmachine interface. These profiles are then replicated on the tele-operated robot in real-time. The 
strong correlation between muscle activation and impedance can be leveraged by using sEMG signals to estimate arm and hand position and impedance. For the latter case, this approach is intuitive since it is well known that muscle force increases with muscular activity [131]. Furthermore, individual muscle forces contribute to net joint torque, whereas their co-activation can modulate joint impedance [132]. However, the extraction of position from muscle forces requires the application of inverse dynamics [133].

The effectiveness of the tele-impedance approach in replicating the impedance of the proximal arm and lower limb was demonstrated by $[118,130,134]$ who extended the teleimpedance approach to the control of the Pisa/IIT SoftHand and exploited the efficiency and robustness of its soft synergy-based design. These authors developed a novel active impedance controller that incorporates both hand stiffness and postural synergy references in real-time. With the goal of exploiting the minimum number of muscles necessary for decoding the reference profiles, in [118] the authors used the major finger antagonist muscle pair - the m. extensor digitorum communis (EDC) and $\mathrm{m}$. flexor digitorum superficialis (FDS) - and two functions of a modified hyperbolic tangent shape [135] to map the reference commands extracted from experimental data. Through tele-impedance control and exploiting two tactile interfaces, which conveyed information about grasping forces and high-frequency texture information to the user, subjects could improve grasp robustness and interaction performance while controlling the Pisa/IIT SoftHand through a myoelectric interface.

These findings offer a novel perspective for the control of prosthetic devices with multiDOFs. The current challenge is to determine whether and in what conditions tele-impedance is a better solution with respect to multi-sEMG signal pattern regression. To address this issue, the choice of the end effector has to be taken into account. Specifically, the question is whether or 
not synergy-based robotic hands should be used, which can cope motor redundancy through their mechatronic design. Additional factors to consider are the extent to which synergy-based designs improve artificial hands' reaction to external disturbances, and/or improve the intuitiveness with which humans can operate them.

\section{Hand synergies: Sensing}

In this section, we consider the dual aspect of actuation, i.e., the sensing domain. Here we describe how "dimensionality reduction" through synergies is not limited to the motor domain, as it also appears to operate in the sensing domain by reducing the huge amount of inputs from sensory receptors into a low-dimensional set of manageable perceptual representations of the

external world. Furthermore, we leverage the notion that it might be possible to determine a relation between sensory and motor domains, which lays the foundations of the concept we refer to as sensory-motor synergies. As reviewed above for the motor domain, we describe how the physiological principles underlying neural sensing can inspire robotics research and design through simplified approaches to the design of haptic interfaces and sensorization of robotic and human hands with a reduced number of sensors. Applications of such approaches include HRI and sensorimotor rehabilitation. We also describe how robotics can provide useful tools to neuroscience for studying sensorimotor integration.

\subsection{Multi-sensory integration and fusion as the perceptual analogue of motor synergies}

Motor synergies can be regarded as maps between the higher dimensional complexity of the mechanical architecture of the human hand and the lower dimensional control space of the action and performance [3]. In essence, perception can be also regarded as a process for the 
reduction of dimensions and complexity. Color vision provides a clear example of complexity reduction. Indeed, the infinite values of wavelength of the photons characterizing a given light can be mapped into a discrete color by specifying just the three dimensions of hue, saturation and brightness [136]. A similar reduction of dimensionality was also studied in haptics. In [137], the author investigated the number of dimensions that is needed to describe haptic perception from a mechanical point of view. Even if such a number is infinite, human touch-related experience seems to take place in a finite, low dimensional space. In these two examples, both in color vision and touch, the nervous system produces a nearly instantaneous reduction of dimensions to convert a complex problem into a manageable set of computational tasks. Several perceptual illusions can be interpreted as the results of this reduction of the stimulus complexity. Following the same reasoning, the existence of a map between the higher-dimensional space of elemental sensory variables involved in the mechanics of touch and the lower dimensional space of perceptual primitives was postulated in [103]. The elements of this mapping can be regarded as projections of the tactual perception manifold onto constrained subspaces, the latter ones individuating increasingly refined approximations of the full spectrum of haptic information. The authors referred to these maps as sensory synergy basis. From a geometrical perspective, this is the counterpart of postural synergy basis in the motor domain [11].

The tactile system uses different strategies to achieve a reduction of stimulus complexity (i.e., a reduction of the perceptual dimensionality). Selective attention on salient features is an example of efficient complexity reduction. As demonstrated in [138], in haptic search tasks the observer focuses on specific features of the stimuli. An object having a salient feature would quickly pop out during the search task, thus reducing the exploration time. With regard to softness discrimination, a possible reduction of dynamic, force-varying tactile information 
operated by the nervous system can be described by a tactile flow paradigm [139], which suggests that, in dynamic conditions, a large part of contact sensing on the finger pad can be described by the flow of strain energy density (SED). Moreover, the integral version of the tactile flow equation can be used to explain the Contact Area Spread Rate (CASR) experimental observation. This finding reveals that a considerable part of tactile ability in object softness discrimination is retained in the relationship between the contact area growth over an indenting probe (e.g. the finger pad which presses the object) and the indenting force itself [140]. These suitable approximations and reductions of haptic information manifold can suggest new strategies to build haptic interfaces, in order to implement trade-offs between accuracy in stimulus rendering and simplicity. For example, recognizing that a simple force-area relation describes a large amount of cutaneous information involved in softness discrimination by probing has inspired the development of simpler and more effective haptic displays for humancomputer interaction (e.g., [140-143]).

Cutaneous cues appear to be more informative than kinaesthetic cues in softness discrimination [144,145]. Applying the "synergy as a basis" description, in [103] the authors ventured to consider kinaesthesia and CASR as the two main "synergies" in softness discrimination: this idea seemed to be further supported by the experimental outcomes in [146], which offered an interesting parallel with synergies in the motor domain. Similarly to the motor domain, performance in softness discrimination increases with the number of sensory synergies involved, i.e. when both kinaesthetic and cutaneous cues are available, even if cutaneous perception provides a more refined "inner representation of object softness". Similar findings have also been reported by length perception experiments [147]. 
Such an increase in perceptual/representation fidelity can be found also in the artificial side. Tactile displays, which provide specific stimuli through skin deformation, are able to elicit a better softness perception than their purely kinesthetic counterparts, i.e., devices that act as force displays. However, the fidelity by which softness can be artificially rendered increases with the number of synergies employed in rendering., i.e., by suitably integrating both types of devices [146].

At the human side, the integration of sensory information occurs both over space and time. An example of temporal integration is when we slide the fingertip over the surface of an object to feel its texture, or we follow a contour to perceive its shape [148]. Spatial integration occurs when information from different body locations is combined. In [149] the authors investigated whether different ways of spatial integration lead to the same or different perceptions of length, by comparing different ways of discriminating length using two fingers, either of the same hand (thumb and index finger) or of the different hands (both index fingers). Results showed that the unimanual and the bimanual grasping conditions yielded best performance. Indeed, in all conditions information from different locations had to be integrated, but apparently this is easiest if body locations are strongly connected. Different types of information can be also jointly exploited to provide a unified perception of tactile properties of the external environment. In [149], the authors investigated whether local curvature can be also used for length discrimination and thus whether extent and curvature information can be integrated. Results showed that when length and curvature were both present in the stimuli, performance (i.e., sensitivity) was significantly better than when just length or curvature alone was present. This provides clear evidence that curvature and length information can be integrated 
in perception. Of these two conditions, the one in which curvature and length correlated as in circular stimuli led to best performance.

Multisensory fusion can be also viewed as an instance of sensory dimensionality reduction. Whenever we explore an object, for example a pencil, we fuse the multiple cues provided by our senses (such as the visual and tactile estimate of its length, color, softness, etc.) in a unique, coherent object representation. Multisensory integration has also an interesting counterpart in robotics, for example for the control of mobile robots [150]. Due to this mechanism of dimensionality reduction, questions like "Is the visual length of the pencil longer than its tactile length?" would sound as meaningless in most of daily life experience (except for philosophical debate), since the redundant estimates gathered from the different sensory channels are merged in a unique multisensory estimate. ${ }^{2}$ What would be an optimal strategy for sensory fusion? We know from classical studies in psychophysics that the level of noise could be considerably different between different sensory channels. In this case, a weighted average of different cues, where the weight of each cue is an inverse function of its noise, would provide a statistically optimal solution to the problem [151]. In their classical paper, Ernst and Banks [152] showed that the actual combination of vision and touch is statistically optimal.

An important related research topic in haptics is the sensory fusion of proprioception and cutaneous touch. Indeed, whenever we move the hand and we contact an external object, the somatosensory system receives multiple afferent signals from the musculoskeletal system (e.g., $[153,154]$ ), and the skin (e.g., [139,155-157]). Our brain fuses these signals to produce a unique and coherent representation of the hands position, contact orientation and motion. Accordingly,

\footnotetext{
${ }^{2}$ Notice however that questions like that are often posed in psychophysical experiments, where specific setups allow to disentangle the cues; see for example [152].
} 
in [158] the authors showed that the perception of motion of a touched surface arises from the integration of tactile cues, proprioceptive cues, and prior assumptions on surface motion state.

As already pointed out, a simple solution to fuse different sensory measurements (i.e. different sensory cues) comprises computing their weighted average [151]. When the weights are chosen as inversely proportional to the relative precision of the measurements, this guarantees a statistically optimal estimate [152]. For signals to be integrated, however, they have to be received in commensurable units of measure. The issue of combining qualitatively different cues was first studied in visual depth perception [151]. The authors distinguished between absolute and relative depth cues. The latter need to be calibrated in a process known as cue promotion in a way that the units become commensurable. Only after cue promotion, the absolute and relative cues can be integrated into an object estimate. A similar model also accounted for the fusion of proprioception and touch in the perception of finger displacement [159]. Moving the finger to contact an external surface, it produces a spread, from an initial point, of the area of contact between the skin and the object. This is even more appreciable if the external object is compliant; in this case, the size of the area increases even further with the finger indentation. Therefore, the spread of the area of contact provides a relative cue to finger displacement or indentation. Accordingly modulating the size of the area of contact through the softness-rendering device illustrated in [143], induces an illusory displacement of the finger. The size of the area of contact is a promoted displacement cue, which is combined with absolute cues, for example from proprioception, to gather a fused estimate of the finger displacement.

A similar fusion of the proprioceptive and the tactile cue might also occur when sliding our finger over a rigid surface and we have to estimate the motion path or the velocity. Touch is able to provide displacement information, showing that the human brain is able to integrate the 
motion signal over time. Three behavioral experiments [160] showed that this is actually the case, that is, observers were able to integrate tactile velocity over time to produce a reliable estimate of the motion path. Using the tactile device described in [161], the authors generated slip motion along several triangular paths. Participants contacted the moving surface with the index fingertip, while keeping the hand world-stationary and they were able to accurately indicate the length and the shape generated by the motion path. The experimental paradigm required estimating the motion path through a mechanism of path integration; heuristics solely based on motion duration would not account for the actual responses. The above-discussed studies showed that, when both proprioceptive and tactile motion cues are available, the somatosensory system fuses these cues in a unique representation of limb motion, thus producing a reduction in signal dimensionality and complexity.

The integration of multiple cues might in principle include information from our motor plan and the predictions that a given action would have on proprioception and touch. This would suggest the possibility of a mapping or correspondence between the strain pattern on the skin and muscle and the coordinated muscle activity, particular for a given action. This mapping can be defined as a sensorimotor synergy [103] and could simplify the interpretation of the complex sensory information from touch. In the next section, we propose a novel perspective on the correlation between sensory and motor signals, and how sensory-motor synergies can be exploited at the neural level to build a manageable perceptual representation. The duality of sensory and motor domains will be also discussed in the context of artificial sensory and motor domains, with applications to sensorization of human hands. 


\subsection{Sensory-motor synergies at the neural level and robotic applications}

The execution of purposeful movements is dependent on a brain-body relationship and a sense of the relationship between ourselves and the environment. The brain can rely on hundreds of thousands of sensors in skin, muscles and joints as well as direct control of hundreds of muscles. Evolution has established a number of predefined structures in the CNS, but how the sensors find their appropriate target neurons and how this process leads to the emergence of meaningful movements among the very large number of DOFs of the body is not known. However, a number of basic principles of the architecture or circuitry structure of the brain are known, as are the activation properties of the sensors. In combination with behavioral analysis of movement patterns and their statistics in humans (e.g., $[11,12,162])$ and animal models (e.g., $[29,163])$, it is slowly becoming possible to model the neural circuitry mechanisms for taskspecific dimensionality reduction in sensorimotor functions, and even how these mechanisms can be learned and established in brain circuitry. The processing is massively parallel, where most tasks of movement control can be expected to engage large proportions of the circuitry. This has the interesting consequence that the same circuitry components are involved in most or all tasks, implying that the brain has in addition solved the challenge of having the solutions to a very large number of motor tasks in the same circuitry components.

However, the modeling of the control circuitry requires a reality check of the viability of the underlying fundamental principles - in this respect, principles from robotics may provide critical insights. One example of such insights is the enabling constraints provided by the 'anatomy' of robotic hand [109], where the mechanics will preferentially allow certain types of movements, but modifications of those movement patterns can be added as needed. In fact, the basic plan of the central nervous systems is similar across such widely different mammals as the 
mouse and the whale [164], but the mechanics of the bodies are not. Therefore, it has been proposed that the biomechanics of our bodies are an important initial factor behind the early organization of the neural control circuitry, for both motor control and sensory processing [165]. But what would be the circuitry mechanisms that support the acquisition of such complex skills?

A possible answer is that the basic mechanisms are not particularly complex, but the learning occurs in many different phases and layers of control in sequence. Perhaps the most easily graspable solution is that of acquisition of sensory synergies, as the learning of (relatively) pure sensory synergies probably occurs in one layer only. This framework provides a demonstration-of-principle that is likely to apply also for the acquisition of muscle synergies for dimensionality reduction during movement. In essence, the skin of our bodies have thousands of sensors [160] that provide monosynaptic (direct synaptic) input to the neurons of the cuneate nucleus in the brainstem. All tactile inputs that reach the neocortex of the brain must first pass the cuneate nucleus where information is integrated by a monolayer of neurons, supported by a limited population of local inhibitory interneurons. Each neuron of the monolayer receives input from hundreds or thousands of sensors. However, in an intracellular analysis of such neurons, the number of primary sensory afferents that provided synapses of substantial influence on the receiving neuron was as few as 5-8 [166], which suggested the involvement of a learning process strengthening a few of the synapses while reducing the weight of other synapses. This observation raised the question of what purpose this learning process might have. Specifically, what was the common denominator that made some sensors provide strong synaptic input to certain cuneate neurons, whereas the efficacy of synaptic input of the same sensors to other neurons was reduced? 
A recent study, in which a novel view on the organization of somatosensory processing in the brain was tested, seems to have shed light on these issues. The study was made possible by likewise recent advances in psychophysics/robotics where a haptic interface designed to explore haptic illusions in humans had been developed [157]. The novel idea being tested was that the somatosensory functions of the brain are based on the fundamental input features defined by contact mechanics [137] rather than the classical neuroscience concepts of receptive fields and sub-modalities. Thus, the individual cuneate neurons did indeed seem to be apt at segregating the input features, or haptic input dimensions [157,167]. A consequence of this phenomenon is that each cuneate neuron has a unique input profile, but the population of cuneate neurons can give precise information about the contact mechanics of a skin-object interaction at any point on the skin. As the input features run across multiple receptive fields, where 100's of sensors are located, whereas the individual cuneate neurons appear to sample information primarily from 5-8 sensors, this is a prime example of a sensory synergy or dimensionality reduction where the astronomic number of combinatorial possibilities provided by the sheet of sensors is reduced to a mere subset by learning mechanisms at the level of the cuneate neurons.

At the neural level, the subsets of sensors identified through the learning process are then most likely selected by the movement statistics and the sensor locations of the individual, where the movement of the skin against objects by the laws of contact mechanics will determine which sensors will be most frequently co-activated. These co-activated sensors will be the ones that become strengthened in their synapses on the cuneate neurons, and the higher the number of cuneate neurons, the higher the number of co-activated sensor sub-groups can be afforded to be represented, and the richer the sensory experience. It is likely that we can think of the organization of the motor control systems in a similar fashion: the movement statistics, which 
initially are determined by the biomechanics of the individual's body, will be laid down in the circuitry structure by learning processes. However, the motor control systems are more complicated than the sensory system reviewed above, as they are subdivided into several layers of varying intrinsic complexity. The innermost control loops reside in the spinal cord, and the earliest muscle synergies are probably established there, perhaps as early as in fetal life. The spinal circuitry is relatively complex, as it is driven in part by motor commands from higher centers and in part from sensory feedback of different types, where the tactile sensors described above are but one of at least three major classes of sensory feedback [168]. More complex synergies are likely dependent on the functionality added by the cerebellum $[8,169]$, which regulates the spatiotemporal structure of the motor commands descending to the spinal circuitry from higher centers.

The strong relationship between the motoric and sensory side can be exploited also in the artificial side, offering useful hints to robotic research. As previously pointed out, the concept of motor synergies enables to reduce the number of DOFs to be enrolled according to a given level of approximation of the motor act and, as extensively discussed, was used in robotics under a controllability point of view, to design and control artificial hands with a reduced number of inputs/actuators.

However, this result can also have another interpretation, under the observability point of view: the question is whether it is possible to reduce the number of independent hand joints to be measured or sensed to achieve hand pose estimation at a given level of approximation. Indeed, if the human hand moves according to patterns of most frequent use, it could be possible to exploit this information to improve hand pose reconstruction despite measurements, which are in general noisy and reduced in number. 
In [170], this approach was applied to whole hand avatar animation, considering only two contact points for the thumb and the index with a haptic interface. Exploiting synergistic information, the forces necessary to track real finger trajectories through virtual springs (between the avatar's tips and the corresponding operator's tips) were transformed in the synergy-space of general forces. The latter ones were subsequently used to estimate the updated synergy variables and then to characterize the whole-hand configuration.

In [171], authors examined the problem of optimal estimation of the posture of a human hand using non-ideal hand pose reconstruction (HPR) systems, or gloves, with many applications in the fields of human-robot interaction, rehabilitation and virtual reality. Indeed, all HPR methods are inherently affected by non-idealities, which limit their performance, such as the complexity of the human hand biomechanics and costs. All these factors usually determine an incomplete and imperfect relation between the measurements and the anatomical DOFs of the human hand. The solution proposed in [171] was to increase the accuracy of the pose reconstruction without modifying the glove hardware - hence basically at no extra cost - by collecting, organizing, and exploiting information on the probabilistic distribution of human hand poses in common tasks. Such information was organized in a database as in [11], represented in a hierarchy of correlation patterns (or postural synergies), and finally fused with glove measurements in a consistent manner. Thus, it was possible to provide good hand pose reconstructions in spite of insufficient and inaccurate sensing data, as demonstrated through simulations and experiments with a low-cost glove. In the companion paper [172], authors pushed forward this analysis, investigating how and where to place sensors on a glove, to get maximum information about the actual hand posture. This problem becomes particularly crucial when constraints limit the number and the quality of the sensors. 
The example of the human hand can again provide inspiration to solve this problem. Indeed, looking at the dorsal skin of the human hand, a non-uniform distribution of different types of mechanoreceptors can be observed. Such receptors were proven to contribute to the kinesthetic perception of finger position (see e.g. [153]). These observations suggest that a nonuniform map of sensitivities to joint angles may exist in the human hand sensory system. Even if the functional role of this architecture remains to be understood, a fascinating explanation might be related to the different importance of distinct elementary percepts in building an overall representation of the hand pose. All these biological data motivated the search for a preferential distribution and density of different typologies of sensors in sensing gloves, with the goal of optimizing their accuracy. This optimal sensor distribution and density, which enables to get maximum information about the actual hand posture, was shaped on the basis of postural synergistic information as represented in [171].

Of particular significance is the mutual inspiration between neuroscience and robotics. Specifically, the kinematic synergy concept, together with the observations on the biology of human mechanoreceptors, was the leading idea for the optimal estimation approach with HPR systems. At the same time, experimental results obtained with artificial devices might further inspire biological investigations, thereby providing theoretical and technical tools to advance the study of human hand sensorimotor apparatus.

\section{Open questions and directions for future research}

This article has reviewed the main scientific findings on the synergistic organization of human sensory-motor apparatus and how this organization has inspired novel paradigms for the development of "'intelligent "' artificial systems. Under this light, the words pronounced by the 
anthropologist Sherwood Washburn [173] more than 50 years ago, “...the modern human brain came after the hominide hand...", not only appeared as an astonishing assertion, but represented the historical starting point for the interdisciplinary reflections we have discussed in this paper. This assumption was linked to the idea that in order to completely understand intelligence, we must first understand the details of sensorimotor system underlying the control of our own hands. We have shown that in robotics it is quite tempting to think of a similar hypothesis, namely that the design of mechanical hands, including the principles of low-level sensing and control, will shape, at least in part, the development of the field of artificial intelligence at large.

The fundamental idea underlying this approach and the related cross-disciplinary work reported in this survey was that (rephrasing Galileo) "the hand and the physical embodiment, which determines its behavior and cognitive capabilities, speaks a language whose words are the sensorimotor synergies", and that only the understanding of this language will enable us to build artificial systems that can have a dialogue with the human counterpart.

These observations motivated questions that were addressed by "THE Hand Embodied" project, however some important scientific questions remain open and should be targeted by future research. These concern the reciprocal linkages between the physical hand and its highlevel control functions, and the way that embodiment enables and determines behaviors and cognitive functions. According to the methodology delineated in this paper, the study of the intrinsic relationship between the hand as a cognitive abstraction and its bodily instance might be furthered by performing neuroscientific and perceptual behavioral studies with participants engaged in controlled manual activities. In parallel, the development of a theoretical framework explaining the underlying neurobiological mechanisms can lay the foundations for the design and control of robotic hands, haptic interfaces, and neuro-prosthetic devices. We deem these studies 
to be necessary to eventually come to an understanding of how the embodied characteristics of the human hand and its sensors, the sensorimotor transformations, and the very constraints they impose, affect and determine the learning and control strategies we use for such fundamental cognitive functions such as exploring, grasping and manipulating.

The multidisciplinary approach we have undertaken shows that human data and hypothesis-driven simulations can be analyzed and used to derive novel system architectures for the "hand" as a cognitive organ, and this knowledge can eventually be applied to design and control robotic devices with superior robustness and flexibility. The program is fundamentally based on the conceptual structure and the geometry of "synergies", and in the broad definition we have used of "enabling constraints": correlations in redundant hand mobility (motor synergies), correlations in redundant cutaneous and kinaesthetic receptors readings (multi-cue integration), and overall sensorimotor system synergies. We believe that these concepts are also keys for advancing the state of the art in artificial systems as well as the creation of the next generation of humanoid robots, advanced haptic systems, and highly integrated neuro-prosthetic devices.

From a technological perspective, work reported in this survey also has important implications for future research. First, the technology of "soft hands" that was adopted in the project THE can be applied in many forms - e.g. using continuum robotics as presented in [174]. Indeed, the use of compliant hands to replace traditional rigid robotic devices opens new opportunities, but also new challenges, in robotic manipulation. Manipulation with a simple, soft-synergy based hand allows for, and indeed requires strong interactions with the environment, including the manipulandum. Through the observation of human hand operations, it can be easily realized how fundamental in everyday grasping the role of hand compliance to 
adapt fingers to the shape of the surrounding objects is. Since the shape of soft hands is determined by the forces exchanged with the objects in contact, objects and environmental constraints can be profitably exploited to functionally shape the hand and to go beyond its nominal kinematic limits by exploiting structural softness $[175,176]$, substantially changing the classical manipulation planning paradigm. Due to their simplicity, compliance and robustness, hands developed following the soft synergy approach represent an ideal platform for the development of novel prostheses, thus opening interesting perspectives in robotic rehabilitation. Initial work using the Pisa/IIT SoftHand as a prosthesis on a number of normal subjects and amputees has provided promising albeit preliminary results in terms of ease of use, intuitive command, grasping and manipulation performance [111]. Furthermore, the exploitation of teleimpedance and EMG-based applications as well as the usage of wearable haptic interfaces can enable a more natural and intuitive control of (synergy-inspired) prosthetic hands [118].

On the other side, considering sensing and tactile-related information feedback, the correct understanding of mechanisms of multi-cue integration and sensor fusion, in other terms of sensory synergies, can not only inspire the design of novel, simple yet effective sensing systems and haptic devices but offer novel insights in a wide range of robotic applications, e.g. mobile robotics. In these applications, where multiple information sources are unified in a unique coherent perceptual representation, synergistic inspiration can be used to enable robotic systems with advanced perceptual and cognitive capabilities. Furthermore, the identification of the most informative forms of sensed data, together with the study of transparent manners to convey them back to the user, can represent the successful approach to improve prosthetic devices, closing the loop between action and perception, and endowing prostheses not only with advanced control systems but also with perceptual properties. 
The challenging goal is to identify trade-offs between simplicity, intuitiveness and effectiveness. Suitably balancing these ingredients is not an easy task: "working hard to make it simple" can be the novel motto for synergy-inspired robotic research. In other terms, getting close to the astonishing capabilities of CNS in producing nearly instantaneous simplifications of the huge amount of human sensory-motor data can be the key for the design of a novel generation of cognition-enabled robotic devices.

However, this road is still largely unexplored and the challenges it poses become more and more intriguing as research on the human hand sensory-motor apparatus provides novel insights. The approach we propose to profitably proceed along this road is to make technological developments and biological research working together in a synergistic fashion. The ultimate goal of this research is to bridge the gap between neuroscience and robotics with the twofold goal of increase the comprehension of the functional and neuroanatomical organization of the human hand, also thanks to technological tools to perform neuroscientific investigations and robotic inspiration, and to derive biology-inspired guidelines for a more effective development of robotic systems, through the usage of mathematical language. 


\section{Acknowledgments}

This work has been partially supported by the European Commission with Collaborative Project no. 248587, 'THE Hand Embodied', within the FP7-ICT-2009-4-2-1 programme 'Cognitive Systems and Robotics', by the European Research Council under the Advanced Grant no.

291166 ,'SoftHands: A Theory of Soft Synergies for a New Generation of Artificial Hands', and by the National Science Foundation grant BCS-1455866 "Collaborative Research: Sensorimotor Control of Hand-Object Interactions". 


\section{References}

[1] Elliott JM, Connolly KJ. A classification of manipulative hand movements. Dev Med Child Neurol 1984;26:283-96. doi:10.1111/j.1469-8749.1984.tb04445.x.

[2] Turvey MT. Action and perception at the level of synergies. Hum Mov Sci 2007;26:657-97. doi:10.1016/j.humov.2007.04.002.

[3] Latash ML. Synergy. New York: Oxford University Press; 2008.

[4] Ting LH, McKay JL. Neuromechanics of muscle synergies for posture and movement. Curr Opin Neurobiol 2007;17:622-8. doi:10.1016/j.conb.2008.01.002.

[5] Bizzi E, Cheung VCK, D’Avella A. Combining modules for movement. Brain Res Rev 2008;57:125-33. doi:doi:10.1016/j.brainresrev.2007.08.004.

[6] Santello M, Lang CE. Are movement disorders and sensorimotor injuries pathologic synergies? When normal multi-joint movement synergies become pathologic. Front Hum Neurosci 2014;8:1050. doi:10.3389/fnhum.2014.01050.

[7] Bizzi E, Cheung VCK. The neural origin of muscle synergies. Front Comput Neurosci 2013;7. doi:10.3389/fncom.2013.00051.

[8] Santello M, Baud-Bovy G, Jörntell H. Neural bases of hand synergies. Front Comput Neurosci 2013;7:23. doi:10.3389/fncom.2013.00023.

[9] Santello M. Synergistic Control of Hand Muscles Through Common Neural Input. Underst. Hum. hand Adv. Robot. Manip., 2014, p. 23-48. doi:10.1007/978-3-319-03017-3_2.

[10] d'Avella A, Lacquaniti F. Control of reaching movements by muscle synergy combinations. Front Comput Neurosci 2013;7. doi:10.3389/fncom.2013.00042.

[11] Santello M, Flanders M, Soechting JF. Postural hand synergies for tool use. J Neurosci 1998;18:10105-15. doi:citeulike-article-id:423192.

[12] Santello M, Flanders M, Soechting JF. Patterns of hand motion during grasping and the influence of sensory guidance. J Neurosci 2002;22:1426-35.

[13] Mason CR, Gomez JE, Ebner TJ. Hand synergies during reach-to-grasp. J Neurophysiol 2001;86:2896-910.

[14] Zatsiorsky VM, Latash ML. Prehension synergies. Exerc Sport Sci Rev 2004;32:75-80. doi:10.1007/s00221-011-2799-4.

[15] Cheung VCK, Turolla A, Agostini M, Silvoni S, Bennis C, Kasi P, et al. Muscle synergy patterns as physiological markers of motor cortical damage. Proc Natl Acad Sci 2012;109:14652-6. doi:10.1073/pnas.1212056109.

[16] Roh J, Rymer WZ, Perreault EJ, Yoo SB, Beer RF. Alterations in upper limb muscle synergy structure in chronic stroke survivors. J Neurophysiol 2013;109:768-81. doi:10.1152/jn.00670.2012.

[17] Brown CY, Asada HH. Inter-finger coordination and postural synergies in robot hands via mechanical implementation of principal component analysis. IEEE-RAS Int. Conf. Intell. Robot. Syst., 2007, p. 2877-82. doi:doi: 10.1109/IROS.2007.4399547.

[18] Ciocarlie MT, Goldfeder C, Allen PK. Dimensionality reduction for hand-independent dexterous robotic grasping. Intell. Robot. Syst. 2007. IROS 2007. IEEE/RSJ Int. Conf., 2007, p. 3270-5. 
doi:10.1109/IROS.2007.4399227.

[19] Napier JR. The prehensile movements of the human hand. J Bone Joint Surg Br 1956;38-B:90213.

[20] Schieber MH, Santello M. Hand function: neural control and peripheral limits to performance. J Appl Physiol 2004;96:2293-300.

[21] Schieber MH, Gardinier J, Liu J. Tension distribution to the five digits of the hand by neuromuscular compartments in the macaque flexor digitorum profundus. J Neurosci 2001;21:2150-8.

[22] Tresch MC, Jarc A. The case for and against muscle synergies. Curr Opin Neurobiol 2009;19:6017. doi:10.1016/j.conb.2009.09.002.

[23] Winges SA, Santello M. Common input to motor units of digit flexors during multi-digit grasping. J Neurophysiol 2004;92:3210-20. doi:10.1152/jn.00516.2004.

[24] Johnston JA, Winges SA, Santello M. Periodic modulation of motor-unit activity in extrinsic hand muscles during multidigit grasping. J Neurophysiol 2005;94:206-18. doi:10.1152/jn.01134.2004.

[25] Winges SA, Kornatz KW, Santello M. Common input to motor units of intrinsic and extrinsic hand muscles during two-digit object hold. J Neurophysiol 2008;99:1119-26. doi:10.1152/jn.01059.2007.

[26] Winges SA, Johnston JA, Santello M. Muscle-pair specific distribution and grip-type modulation of neural common input to extrinsic digit flexors. J Neurophysiol 2006;93:1258-66. doi:10.1152/jn.00327.2006.

[27] Poston B, Danna-Dos Santos A, Jesunathadas M, Hamm TM, Santello M. Force-independent distribution of correlated neural inputs to hand muscles during three-digit grasping. J Neurophysiol 2010;104:1141-54.

[28] Brochier T, Spinks RL, Umilta MA, Lemon RN. Patterns of muscle activity underlying objectspecific grasp by the macaque monkey. J Neurophysiol 2004;92:1770-82. doi:10.1152/jn.00976.2003.

[29] Overduin SA, D’Avella A, Roh J, Bizzi E. Modulation of muscle synergy recruitment in primate grasping. J Neurosci 2008;28:880-92. doi:10.1523/JNEUROSCI.2869-07.2008.

[30] Weiss EJ, Flanders M. Muscular and postural synergies of the human hand. J Neurophysiol 2004;92:523-35. doi:10.1152/jn.01265.2003.

[31] Breteler MDK, Simura KJ, Flanders M. Timing of muscle activation in a hand movement sequence. Cereb Cortex 2007;17:803-15. doi:10.1093/cercor/bhk033.

[32] Tagliabue M, Ciancio AL, Brochier T, Eskiizmirliler S, Maier MA. Differences between kinematic synergies and muscle synergies during two-digit grasping. Front Hum Neurosci 2015;9:165. doi:10.3389/fnhum.2015.00165.

[33] Gordon AM. Development of hand motor control. In: Kalverboer A, editor. Handb. brain Behav. Hum. Dev., Kluwer Acad. Publ.; 2001, p. 513-37.

[34] Ingram JN, Körding KP, Howard IS, Wolpert DM. The statistics of natural hand movements. Exp Brain Res 2008;188:223-36. doi:10.1007/s00221-008-1355-3.

[35] Thakur PH, Bastian AJ, Hsiao SS. Multidigit movement synergies of the human hand in an unconstrained haptic exploration task. J Neurosci 2008;28:1271-81. 
[36] Vinjamuri R, Sun M, Weber D, Wang W, Crammond D, Mao ZH. Quantizing and characterizing the variance of hand postures in a novel transformation task. Proc 31st Annu Int Conf IEEE Eng Med Biol Soc Eng Futur Biomed EMBC 2009 2009:5312-5.

[37] Latash ML, Friedman J, Kim SW, Feldman AG, Zatsiorsky VM. Prehension synergies and control with referent hand configurations. Exp Brain Res 2010;202:213-29. doi:10.1007/s00221-0092128-3.

[38] Latash ML. Motor synergies and the equilibrium-point hypothesis. Motor Control 2010;14:294322. doi:10.1016/j.bbi.2008.05.010.

[39] Latash ML, Scholz JP, Schoner G. Toward a new theory of motor synergies. Motor Control 2007;11:276-308. doi:17715460.

[40] Gentner R, Classen J. Modular organization of finger movements by the human central nervous system. Neuron 2006;52:731-42. doi:10.1016/j.neuron.2006.09.038.

[41] Overduin SA, D’Avella A, Carmena JM, Bizzi E. Microstimulation activates a handful of muscle synergies. Neuron 2012;76:1071-7. doi:10.1016/j.neuron.2012.10.018.

[42] Desmurget M, Richard N, Harquel S, Baraduc P, Szathmari A, Mottolese C, et al. Neural representations of ethologically relevant hand/mouth synergies in the human precentral gyrus. Proc Natl Acad Sci 2014;111:5718-22. doi:10.1073/pnas.1321909111.

[43] Castiello U. The neuroscience of grasping. Nat Rev Neurosci 2005;6:726-36. doi:doi:10.1038/nrn1744.

[44] Gallivan JP, McLean DA, Valyear KF, Culham JC. Decoding the neural mechanisms of human tool use. Elife 2013:2:e00425. doi:10.7554/eLife.00425.001.

[45] Vingerhoets G. Contribution of the posterior parietal cortex in reaching, grasping, and using objects and tools. Front Psychol 2014;5:1-17. doi:10.3389/fpsyg.2014.00151.

[46] Handjaras G, Bernardi G, Benuzzi F, Nichelli P, Pietrini P, Ricciardi E. A topographical organization for action representation in the human brain. Hum Brain Mapp 2015;36:3832-44.

[47] Fleming G. The Motor Cortex in Man in the Light of Hughlings Jackson's Doctrines.(Brain, vol. lix, p. 135, June, 1936.) Foerster, O. Br J Psychiatry 1936;82:677-8. doi:10.1192/bjp.82.340.677a.

[48] Felder UB. Sensible Corticale Felder. Bumke Foerster's Handb Der Neurol Berlin, J Springer 1936;6:1-449.

[49] Penfield W, Rasmussen T. The cerebral cortex of man; a clinical study of localization of function. Acad Med 1950;25:375. doi:10.1097/00001888-195009000-00037.

[50] Woolsey CN, Settlage PH, Meyer DR, Sencer W, Hamuy TP, Travis A. Patterns of localization in precentral and" supplementary" motor areas and their relation to the concept of a premotor area. Res Publ - Assoc Res Nerv Ment Dis 1951;30:238-64.

[51] Penfield W, Boldrey E. Somatic motor and sensory representation in the cerebral cortex of man as studied by electrical stimulation. Brain 1937;60:389-443. doi:10.1093/brain/60.4.389.

[52] Schieber MH. Somatotopic gradients in the distributed organization of the human primary motor cortex hand area: evidence from small infarcts. Exp Brain Res Exp Hirnforschung Expérimentation Cérébrale 1999;128:139-48. doi:10.1007/s002210050829.

[53] Schieber MH. Constraints on somatotopic organization in the primary motor cortex. J 
Neurophysiol 2001;86:2125-43.

[54] Hluštík P, Solodkin A, Gullapalli RP, Noll DC, Small SL. Somatotopy in Human Primary Motor and Somatosensory Hand Representations Revisited. Cereb Cortex 2001;11:312-21. doi:10.1093/cercor/11.4.312.

[55] Indovina I, Sanes JN. On somatotopic representation centers for finger movements in human primary motor cortex and supplementary motor area. Neuroimage 2001;13:1027-34. doi:10.1006/nimg.2001.0776.

[56] Dechent P, Frahm J. Functional somatotopy of finger representations in human primary motor cortex. Hum Brain Mapp 2003;18:272-83. doi:10.1002/hbm.10084.

[57] Graziano MSA, Taylor CSR, Moore T. Complex movements evoked by microstimulation of precentral cortex. Neuron 2002;34:841-51. doi:10.1016/S0896-6273(02)00698-0.

[58] Aflalo TN, Graziano MSA. Partial tuning of motor cortex neurons to final posture in a freemoving paradigm. Proc Natl Acad Sci 2006;103:2909-14. doi:10.1073/pnas.0511139103.

[59] Brown AR, Teskey GC. Motor Cortex Is Functionally Organized as a Set of Spatially Distinct Representations for Complex Movements. J Neurosci 2014;34:13574-85. doi:10.1523/JNEUROSCI.2500-14.2014.

[60] Ejaz N, Hamada M, Diedrichsen J. Hand use predicts the structure of representations in sensorimotor cortex. Nat Neurosci 2015;18:1034-40.

[61] Gentner R, Gorges S, Weise D, Kampe K a., Buttmann M, Classen J. Encoding of motor skill in the corticomuscular system of musicians. Curr Biol 2010;20:1869-74. doi:10.1016/j.cub.2010.09.045.

[62] Leo A, Handjaras G, Bianchi M, Marino H, Gabiccini M, Bicchi A, et al. The "alphabet" of human hand movements: a fMRI study on the neural correlates of postural synergies. Annu. Meet. Organ. Hum. Brain Mapp., 2014.

[63] Leo A, Handjaras G, Bianchi M, Marino H, Gabiccini M, Guidi A, et al. How the brain moves the hand: a synergy-based control is encoded in the human motor cortical areas n.d. doi:(Unpublished: Under Review).

[64] Naselaris T, Kay KN, Nishimoto S, Gallant JL. Encoding and decoding in fMRI. Neuroimage 2011;56:400-10. doi:10.1016/j.neuroimage.2010.07.073.

[65] Varoquaux G, Thirion B. How machine learning is shaping cognitive neuroimaging. Gigascience 2014;3:28. doi:10.1186/2047-217X-3-28.

[66] Agashe HA, Paek AY, Zhang Y, Contreras-Vidal JL. Global cortical activity predicts shape of hand during grasping. Front Neurosci 2015;9:121. doi:10.3389/fnins.2015.00121.

[67] Flint RD, Wang PT, Wright ZA, King CE, Krucoff MO, Schuele SU, et al. Extracting kinetic information from human motor cortical signals. Neuroimage 2014;101:695-703. doi:10.1016/j.neuroimage.2014.07.049.

[68] Pistohl T, Schulze-Bonhage A, Aertsen A, Mehring C, Ball T. Decoding natural grasp types from human ECoG. Neuroimage 2012;59:248-60. doi:10.1016/j.neuroimage.2011.06.084.

[69] Kaas JH, Gharbawie OA, Stepniewska I. Cortical networks for ethologically relevant behaviors in primates. Am J Primatol 2013;75:407-14. doi:10.1002/ajp.22065.

[70] Kirsch E, Rivlis G, Schieber MH. Primary motor cortex neurons during individuated finger and 
wrist movements: correlation of spike firing rates with the motion of individual digits versus their principal components. Front Neurol 2014;5. doi:10.3389/fneur.2014.00070.

[71] Mollazadeh M, Aggarwal V, Thakor NV, Schieber MH. Principal components of hand kinematics and neurophysiological signals in motor cortex during reach to grasp movements. J Neurophysiol 2014;112:1857-70. doi:10.1152/jn.00481.2013.

[72] Soechting JF, Flanders M. Flexibility and repeatability of finger movements during typing: analysis of multiple degrees of freedom. J Comput Neurosci 1997;4:29-46. doi:10.1023/A:1008812426305.

[73] Ciocarlie MT, Allen PK. Hand posture subspaces for dexterous robotic grasping. Int J Rob Res 2009;28:851-67. doi:10.1177/0278364909105606.

[74] Ficuciello F, Palli G, Melchiorri C, Siciliano B. Experimental evaluation of postural synergies during reach to grasp with the UB hand IV. Intell. Robot. Syst. (IROS), 2011 IEEE/RSJ Int. Conf., 2011, p. 1775-80.

[75] Dragan A, Srinivasa S. Integrating human observer inferences into robot motion planning. Auton Robots 2014;37:351-68. doi:10.1007/s10514-014-9408-X.

[76] Bartneck C, Kulić D, Croft E, Zoghbi S. Measurement instruments for the anthropomorphism, animacy, likeability, perceived intelligence, and perceived safety of robots. Int J Soc Robot 2009;1:71-81. doi:10.1007/s12369-008-0001-3.

[77] Duffy B. Anthropomorphism and robotics. Animat. Expressive Characters Soc. Interact., London: 2002.

[78] Riek LD, Rabinowitch TC, Chakrabarti B, Robinson P. How anthropomorphism affects empathy toward robots. Proc. 4th ACM/IEEE Int. Conf. Hum. Robot Interact. - HRI ’09, 2009, p. 245. doi:10.1145/1514095.1514158.

[79] Hauser H, Neumann G, Ijspeert AJ, Maass W. Biologically inspired kinematic synergies enable linear balance control of a humanoid robot. Biol Cybern 2011;104:235-49. doi:10.1007/s00422011-0430-1.

[80] Bootsma RJ, Marteniuk RG, MacKenzie CL, Zaal FTJM. The speed-accuracy trade-off in manual prehension: effects of movement amplitude, object size and object width on kinematic characteristics. Exp Brain Res 1994;98:535-41. doi:10.1007/BF00233990.

[81] Chieffi S, Gentilucci M. Coordination between the transport and the grasp components during prehension movements. Exp Brain Res 1993;94:471-7. doi:10.1007/BF00230205.

[82] Jeannerod M. The timing of natural prehension movements. J Mot Behav 1984;16:235-54. doi:10.1080/00222895.1984.10735319.

[83] Marteniuk RG, Leavitt JL, MacKenzie CL, Athenes S. Functional relationships between grasp and transport components in a prehension task. Hum Mov Sci 1990;9:149-76. doi:10.1016/01679457(90)90025-9.

[84] Paulignan Y, MacKenzie C, Marteniuk R, Jeannerod M. The coupling of arm and finger movements during prehension. Exp Brain Res 1990;79:431-5. doi:10.1016/0022-460X(90)90652G.

[85] Paulignan Y, MacKenzie C. Selective perturbation of visual input during prehension movements. Exp Brain Res 1991;83:502-12. doi:10.1007/BF00231858.

[86] Liarokapis MV, Artemiadis PK, Kyriakopoulos KJ. Task discrimination from myoelectric activity: 
a learning scheme for EMG-based interfaces. Rehabil. Robot. (ICORR), 2013 IEEE Int. Conf., 2013, p. 1-6. doi:10.1109/ICORR.2013.6650366.

[87] Liarokapis MV, Artemiadis PK, Bechlioulis CP, Kyriakopoulos KJ. Directions, Methods and Metrics for Mapping Human to Robot Motion with Functional Anthropomorphism: A Review. 2013.

[88] Liarokapis MV, Artemiadis PK, Kyriakopoulos KJ, Manolakos ES. A learning scheme for reach to grasp movements: On EMG-based interfaces using task specific motion decoding models. Biomed Heal Informatics, IEEE J 2013;17:915-21. doi:10.1109/JBHI.2013.2259594.

[89] Liarokapis MV, Dollar AM, Kyriakopoulos KJ. Humanlike, task-specific reaching and grasping with redundant arms and low-complexity hands. Adv. Robot. (ICAR), 2015 Int. Conf., 2015, p. 490-7. doi:10.1109/ICAR.2015.7251501.

[90] Filippidis IF, Kyriakopoulos KJ, Artemiadis PK. Navigation functions learning from experiments: Application to anthropomorphic grasping. Robot. Autom. (ICRA), 2012 IEEE Int. Conf., 2012, p. 570-5. doi:10.1109/ICRA.2012.6225168.

[91] Peer A, Stanczyk B, Buss M. Haptic telemanipulation with dissimilar kinematics. Proc. IEEE/RSJ Int. Conf. Intell. Robot. Syst., 2005, p. 3493-8. doi:10.1109/IROS.2005.1545349.

[92] Pao L, Speeter TH. Transformation of human hand positions for robotic hand control. Int. Conf. Robot. Autom., 1989, p. 1758-63. doi:10.1109/ROBOT.1989.100229.

[93] Griffin WB, Findley RP, Turner ML, Cutkosky MR. Calibration and mapping of a human hand for dexterous telemanipulation. ASME IMECE 2000 Conf. Haptic Interfaces Virtual Environ. Teleoperator Syst. Symp., 2000.

[94] Gioioso G, Salvietti G, Malvezzi M, Prattichizzo D. Mapping synergies from human to robotic hands with dissimilar kinematics: an approach in the object domain. Robot IEEE Trans 2013;29:825-37. doi:10.1109/TRO.2013.2252251.

[95] Gioioso G, Salvietti G, Malvezzi M, Prattichizzo D. An object-based approach to map human hand synergies onto robotic hands with dissimilar kinematics. Robot. Sci. Syst. VIII, 2013.

[96] Salvietti G, Malvezzi M, Gioioso G, Prattichizzo D. On the use of homogeneous transformations to map human hand movements onto robotic hands. 2014 IEEE Int. Conf. Robot. Autom., IEEE; 2014, p. 5352-7. doi:10.1109/ICRA.2014.6907646.

[97] Salvietti G, Meli L, Gioioso G, Malvezzi M, Prattichizzo D. Object-based bilateral telemanipulation between dissimilar kinematic structures. IEEE/RSJ Int. Conf. Intell. Robot. Syst., 2013, p. 5451-6. doi:10.1109/IROS.2013.6697145.

[98] Salvietti G, Gioioso G, Malvezzi M, Prattichizzo D, Serio A, Farnioli E, et al. HANDS. DVI: A DeVice-Independent programming and control framework for robotic HANDS. Gearing up Accel. cross-fertilization between Acad. Ind. Robot. Res. Eur., Springer International Publishing; 2014, p. 197-215. doi:10.1007/978-3-319-02934-4_10.

[99] Malvezzi M, Gioioso G, Salvietti G, Prattichizzo D. Syngrasp: A matlab toolbox for underactuated and compliant hands. Robot Autom Mag IEEE 2015;PP:1-1. doi:10.1109/MRA.2015.2408772.

[100] Lashley KS. Basic neural mechanisms in behavior. Psychol Rev 1930;37:1-24. doi:10.1037/h0074134.

[101] Fu Q, Hasan Z, Santello M. Transfer of learned manipulation following changes in degrees of freedom. J Neurosci 2011;31:13576-84. doi:10.1523/JNEUROSCI.1143-11.2011. 
[102] Gabiccini M, Bicchi A, Prattichizzo D, Malvezzi M. On the role of hand synergies in the optimal choice of grasping forces. Auton Robots 2011;31:235-52. doi:10.1007/s10514-011-9244-1.

[103] Bicchi A, Gabiccini M, Santello M. Modelling natural and artificial hands with sinergie. Phil Trans R Soc B 2011;366:3153-61.

[104] Prattichizzo D, Malvezzi M, Gabiccini M, Bicchi A. On motion and force controllability of precision grasps with hands actuated by soft synergies. IEEE Trans Robot 2013;29:1440-56. doi:10.1109/TRO.2013.2273849.

[105] Prattichizzo D, Malvezzi M, Gabiccini M, Bicchi A. On the manipulability ellipsoids of underactuated robotic hands with compliance. Rob Auton Syst 2012;60:337-46. doi:http://dx.doi.org/10.1016/j.robot.2011.07.014.

[106] Gabiccini M, Farnioli E, Bicchi A. Grasp analysis tools for synergistic underactuated robotic hands. Int J Rob Res 2013;32:1553-76. doi:10.1177/0278364913504473.

[107] Farnioli E, Gabiccini M, Bonilla M, Bicchi A. Grasp compliance regulation in synergistically controlled robotic hands with vsa. Intell. Robot. Syst. (IROS), 2013 IEEE/RSJ Int. Conf., 2013, p. 3015-22. doi:10.1109/IROS.2013.6696783.

[108] Catalano MG, Grioli G, Serio A, Farnioli E, Piazza C, Bicchi A. Adaptive synergies for a humanoid robot hand. Humanoid Robot. (Humanoids), 2012 12th IEEE-RAS Int. Conf., 2012, p. 7-14. doi:doi: 10.1109/HUMANOIDS.2012.6651492.

[109] Catalano MG, Grioli G, Farnioli E, Serio A, Piazza C, Bicchi A. Adaptive synergies for the design and control of the Pisa/IIT SoftHand. Int J Rob Res 2014;33:768-82. doi:10.1177/0278364913518998.

[110] Gailey A, Godfrey S, Breighner R, Andrews K, Zhao K, Bicchi A, et al. Functional testing of a soft synergy-based artificial prosthetic hand. Soc. Neurosci., 45th Meet. Soc. Neurosci., Chicago IL, USA, Chicago IL, USA: 2015, p. Abst. 249.6461.

[111] Zhao K, Breighner R, Theuer A, Godfrey S., Bianchi M, Catalano MG, et al. Application of a novel robotic hand as a myoelectric prosthetic prototype: Proof of concept in a single patient. ISPO World Congr., 2015.

[112] Muceli S, Jiang N, Farina D. Extracting signals robust to electrode number and shift for online simultaneous and proportional myoelectric control by factorization algorithms. Neural Syst Rehabil Eng IEEE Trans 2014;22:623-33. doi:10.1109/TNSRE.2013.2282898.

[113] Merletti R, Aventaggiato M, Botter A, Holobar A, Marateb H, Vieira TMM. Advances in surface EMG: recent progress in detection and processing techniques. Crit Rev Biomed Eng 2010;38:30545. doi:10.1615/CritRevBiomedEng.v38.i4.10.

[114] Castellini C, van der Smagt P. Evidence of muscle synergies during human grasping. Biol Cybern 2013;107:233-45. doi:10.1007/s00422-013-0548-4.

[115] Jiang N, Englehart K, Parker P. Extracting simultaneous and proportional neural control information for multiple-DOF prostheses from the surface electromyographic signal. Biomed Eng IEEE Trans 2009;56:1070-80. doi:10.1109/TBME.2008.2007967.

[116] Powell MA, Thakor NV. A training strategy for learning pattern recognition control for myoelectric prostheses. J Prosthetics Orthot JPO 2013;25:30-41. doi:10.1097/JPO.0b013e31827af7c1.

[117] Gijsberts A, Bohra R, Sierra González D, Werner A, Nowak M, Caputo B, et al. Stable 
myoelectric control of a hand prosthesis using non-linear incremental learning. Front Neurorobot 2014;8:8. doi:10.3389/fnbot.2014.00008.

[118] Ajoudani A, Godfrey SB, Bianchi M, Catalano MG, Grioli G, Tsagarakis NG, et al. Exploring teleimpedance and tactile feedback for intuitive control of the Pisa/IIT SoftHand. IEEE Trans Haptics 2014;7:203-15. doi:10.1109/TOH.2014.2309142.

[119] Liarokapis MV, Artemiadis PK, Katsiaris PT, Kyriakopoulos KJ, Manolakos ES. Learning human reach-to-grasp strategies: Towards EMG-based control of robotic arm-hand systems. Robot. Autom. (ICRA), 2012 IEEE Int. Conf., 2012, p. 2287-92. doi:10.1109/ICRA.2012.6225047.

[120] Ison M, Artemiadis PK. The role of muscle synergies in myoelectric control: trends and challenges for simultaneous multifunction control. J Neural Eng 2014;11:051001. doi:10.1088/17412560/11/5/051001.

[121] Reilly KT, Mercier C, Schieber MH, Sirigu A. Persistent hand motor commands in the amputees' brain. Brain 2006;129:2211-23. doi:10.1093/brain/awl154.

[122] Tenore FV., Ramos A, Fahmy A, Acharya S, Etienne-Cummings R, Thakor NV. Decoding of individuated finger movements using surface electromyography. Biomed Eng IEEE Trans 2009;56:1427-34. doi:10.1109/TBME.2008.2005485.

[123] Castellini C, Gruppioni E, Davalli A, Sandini G. Fine detection of grasp force and posture by amputees via surface electromyography. J Physiol 2009;103:255-62. doi:doi:10.1016/j.jphysparis.2009.08.008.

[124] Biddiss E, Chau T. Upper-limb prosthetics: critical factors in device abandonment. Am J Phys Med Rehabil 2007;86:977-87. doi:10.1097/PHM.0b013e3181587f6c.

[125] Peerdeman B, Boere D, Witteveen HJB, Huis in 'tVeld MHA, Hermens HJ, Stramigioli S, et al. Myoelectric forearm prostheses: State of the art from a user-centered perspective. J Rehabil Res Dev 2011;48:719. doi:10.1682/JRRD.2010.08.0161.

[126] Ison M, Artemiadis PK. Proportional myoelectric control of robots: muscle synergy development drives performance enhancement, retainment, and generalization. Robot IEEE Trans 2015;31:25968. doi:10.1109/TRO.2015.2395731.

[127] Sierra González D, Castellini C. A realistic implementation of ultrasound imaging as a humanmachine interface for upper-limb amputees. Front Neurorobot 2013;7:17. doi:10.3389/fnbot.2013.00017.

[128] Gomi H, Osu R. Task-dependent viscoelasticity of human multijoint arm and its spatial characteristics for interaction with environments. J Neurosci 1998;18:8965-78.

[129] Ajoudani A, Tsagarakis NG, Bicchi A. Tele-impedance: Towards transferring human impedance regulation skills to robots. Robot. Autom. (ICRA), 2012 IEEE Int. Conf., 2012, p. 382-8. doi:10.1109/ICRA.2012.6224904.

[130] Ajoudani A, Tsagarakis NG, Bicchi A. Tele-impedance: Teleoperation with impedance regulation using a body-machine interface. Int J Rob Res 2012;31:1642-56. doi:10.1177/0278364912464668.

[131] Milner-Brown HS, Stein RB. The relation between the surface electromyogram and muscular force. J Physiol 1975;246:549-69. doi:10.1113/jphysiol.1975.sp010904.

[132] Osu R, Gomi H. Multijoint muscle regulation mechanisms examined by measured human arm stiffness and EMG signals. J Neurophysiol 1999;81:1458-68.

[133] Erdemir A, McLean S, Herzog W, Van den Bogert AJ. Model-based estimation of muscle forces 
exerted during movements. Clin Biomech 2007;22:131-54.

doi:10.1016/j.clinbiomech.2006.09.005.

[134] Karavas N, Ajoudani A, Tsagarakis NG, Saglia J, Bicchi A, Caldwell D. Tele-impedance based stiffness and motion augmentation for a knee exoskeleton device. Robot Autom (ICRA), 2013 IEEE Int Conf 2013:2194-200. doi:10.1109/ICRA.2013.6630872.

[135] Chen CT, Peng ST, Chang WD. A Nonlinear Control Strategy Based on Using a Shape-Tunable Neural Controller. J Chem Eng JAPAN 1997;30:637-46. doi:http://doi.org/10.1252/jcej.30.637.

[136] Palmer SE. Vision science: Photons to phenomenology. Cambridge, MA, USA: MIT Press; 1999.

[137] Hayward V. Is there a "plenhaptic" function? Philos Trans R Soc B Biol Sci 2011;366:3115-22. doi:10.1098/rstb.2011.0150.

[138] Kappers AML, Bergmann Tiest WM. Feature saliency and integration in haptic perception. J Robot Soc Japan 2012;30:456-9. doi:10.7210/jrsj.30.456.

[139] Bicchi A, Scilingo EP, Ricciardi E, Pietrini P. Tactile flow explains haptic counterparts of common visual illusions. Brain Res Bull 2008;75:737-41. doi:10.1016/j.brainresbull.2008.01.011.

[140] Bicchi A, Rossi D De, Scilingo EP. The role of the contact area spread rate in haptic discrimination of softness. Robot Autom IEEE Trans 2000;16:496-504. doi:10.1109/70.880800.

[141] Bianchi M, Serio A, Scilingo EP, Bicchi A. A new fabric-based softness display. 2010 IEEE Haptics Symp., IEEE; 2010, p. 105-12. doi:10.1109/HAPTIC.2010.5444668.

[142] Bianchi M, Scilingo EP, Serio A, Bicchi A. A new softness display based on bi-elastic fabric. EuroHaptics Conf 2009 Symp Haptic Interfaces Virtual Environ Teleoperator Syst World Haptics 2009 Third Jt 2009:382-3. doi:10.1109/WHC.2009.4810905.

[143] Bianchi M, Serio A. Design and Characterization of a Fabric-based Softness Display. Haptics, IEEE Trans 2015;8:152-63. doi:10.1109/TOH.2015.2404353.

[144] Srinivasan MA, LaMotte RH. Tactual discrimination of softness. J Neurophysiol 1995;73:88-101.

[145] Bergmann Tiest WM, Kappers AML. Cues for Haptic Perception of Compliance. IEEE Trans Haptics 2009;2:189-99. doi:10.1109/TOH.2009.16.

[146] Scilingo EP, Bianchi M, Grioli G, Bicchi A. Rendering softness: Integration of kinesthetic and cutaneous information in a haptic device. IEEE Trans Haptics 2010;3:109-18. doi:10.1109/TOH.2010.2.

[147] Bergmann Tiest WM, van der Hoff LMA, Kappers AML. Cutaneous and kinaesthetic perception of traversed distance. World Haptics Conf. (WHC), 2011 IEEE, 2011, p. 593-7. doi:10.1109/WHC.2011.5945552.

[148] Lederman SJ, Klatzky RL. Haptic perception: A tutorial. Attention, Perception, Psychophys 2009;71:1439-59. doi:10.3758/APP.71.7.1439.

[149] Panday V, Bergmann Tiest WM, Kappers AML. Integration of length and curvature in haptic perception. Sci Rep 2014;4:3856. doi:10.1038/srep03856.

[150] Luo RC, Kay MG. The role of multisensor integration and fusion in the operation of mobile robots. IEEE Int Work Intell Robot Tokyo, Japan 1988.

[151] Landy MS, Maloney LT, Johnston EB, Young M. Measurement and modeling of depth cue combination: In defense of weak fusion. Vision Res 1995;35:389-412. doi:10.1016/00426989(94)00176-M. 
[152] Ernst MO, Banks MS. Humans integrate visual and haptic information in a statistically optimal fashion. Nature 2002;415:429-33. doi:10.1038/415429a.

[153] Edin BB, Abbs JH. Finger movement responses of cutaneous mechanoreceptors in the dorsal skin of the human hand. J Neurophysiol 1991;65:657-70.

[154] Proske U, Gandevia SC. The proprioceptive senses: their roles in signaling body shape, body position and movement, and muscle force. Physiol Rev 2012;92:1651-97. doi:10.1152/physrev.00048.2011.

[155] Srinivasan MA, LaMotte RH. Tactile Discrimination of Softness. J Neurophysiol 1995;73:88-101. doi:10.1007/978-3-0348-9016-8_11.

[156] Dallmann CJ, Ernst MO, Moscatelli A. The role of vibration in tactile speed perception. J Neurophysiol 2015;114:3131-9. doi:10.1152/jn.00621.2015.

[157] Hayward V, Terekhov AV, Wong S-C, Geborek P, Bengtsson F, Jörntell H. Spatio-temporal skin strain distributions evoke low variability spike responses in cuneate neurons. J R Soc Interface 2014;11:20131015. doi:10.1098/rsif.2013.1015.

[158] Moscatelli A, Hayward V, Wexler M, Ernst MO. Illusory Tactile Motion Perception: An Analog of the Visual Filehne Illusion. Sci Rep 2015;5:14584. doi:10.1038/srep14584.

[159] Moscatelli A, Bianchi M, Serio A, Al Atassi O, Fani S, Terekhov A, et al. A change in the fingertip contact area induces an illusory displacement of the finger. Eurohaptics 2014, 2014, p. 411. doi:10.1007/978-3-662-44196-1_10.

[160] Moscatelli A, Naceri A, Ernst MO. Path integration in tactile perception of shapes. Behav Brain Res 2014;274:355-64. doi:10.1016/j.bbr.2014.08.025.

[161] Fritschi M, Ernst MO, Buss M. Integration of kinesthetic and tactile display-a modular design concept. Proc. EuroHaptics, 2006, p. 607-12.

[162] Santello M, Soechting JF. Force synergies for multifingered grasping. Exp Brain Res 2000;133:457-67. doi:10.1007/s002210000420.

[163] Mason CR, Theverapperuma LS, Hendrix CM, Ebner TJ. Monkey hand postural synergies during reach-to-grasp in the absence of vision of the hand and object. J Neurophysiol 2004;91:2826-37. doi:10.1152/jn.00653.2003.

[164] Herculano-Houzel S, Manger PR, Kaas JH. Brain scaling in mammalian evolution as a consequence of concerted and mosaic changes in numbers of neurons and average neuronal cell size. Front Neuroanat 2014;8. doi:10.3389/fnana.2014.00077.

[165] Nilsson M, Jorntell H. Biologically Inspired Adaptive Control with Automatic Identification of Sensor Synergies. IEEE Int. Conf. Robot. Autom. (ICRA2013), Work. Hand Synerg., 2013.

[166] Bengtsson F, Brasselet R, Johansson RS, Arleo A, Jörntell H. Integration of sensory quanta in cuneate nucleus neurons in vivo. PLoS One 2013;8:e56630. doi:10.1371/journal.pone.0056630.

[167] Jörntell H, Bengtsson F, Geborek P, Spanne A, Terekhov AV, Hayward V. Segregation of tactile input features in neurons of the cuneate nucleus. Neuron 2014;83:1444-52. doi:10.1016/j.neuron.2014.07.038.

[168] Jankowska E. Interneuronal relay in spinal pathways from proprioceptors. Prog Neurobiol 1992;38:335-78. doi:10.1016/0301-0082(92)90024-9.

[169] Spanne A, Jorntell H. Processing of multi-dimensional sensorimotor information in the spinal and 
cerebellar neuronal circuitry: a new hypothesis. PLoS Comput Biol 2013;9:e1002979. doi:10.1371/journal.pcbi.1002979.

[170] Mulatto S, Formaglio A, Malvezzi M, Prattichizzo D. Using postural synergies to animate a lowdimensional hand avatar in haptic simulation. Haptics, IEEE Trans 2013;6:106-16. doi:10.1109/TOH.2012.13.

[171] Bianchi M, Salaris P, Bicchi A. Synergy-based hand pose sensing: Reconstruction enhancement. Int J Rob Res 2013;32:396-406. doi:10.1177/0278364912474078.

[172] Bianchi M, Salaris P, Bicchi A. Synergy-based hand pose sensing: Optimal glove design. Int J Rob Res 2013;32:407-24. doi:10.1177/0278364912474079.

[173] Washburn SL. Tools and human evolution. Sci Am 1960;203:63-75. doi:10.1038/scientificamerican0960-62.

[174] Deimel R, Brock O. A novel type of compliant, underactuated robotic hand for dexterous grasping. Robot. Sci. Syst., 2014, p. 1687-92.

[175] Eppner C, Brock O. Grasping unknown objects by exploiting shape adaptability and environmental constraints. Intell. Robot. Syst. (IROS), 2013 IEEE/RSJ Int. Conf., 2013, p. 40006. doi:10.1109/IROS.2013.6696928.

[176] Bonilla M, Farnioli E, Piazza C, Catalano MG, Grioli G, Garabini M, et al. Grasping with Soft Hands. Humanoid Robot (Humanoids), 2014 14th IEEE-RAS Int Conf 2014:581-7. doi:10.1109/HUMANOIDS.2014.7041421. 

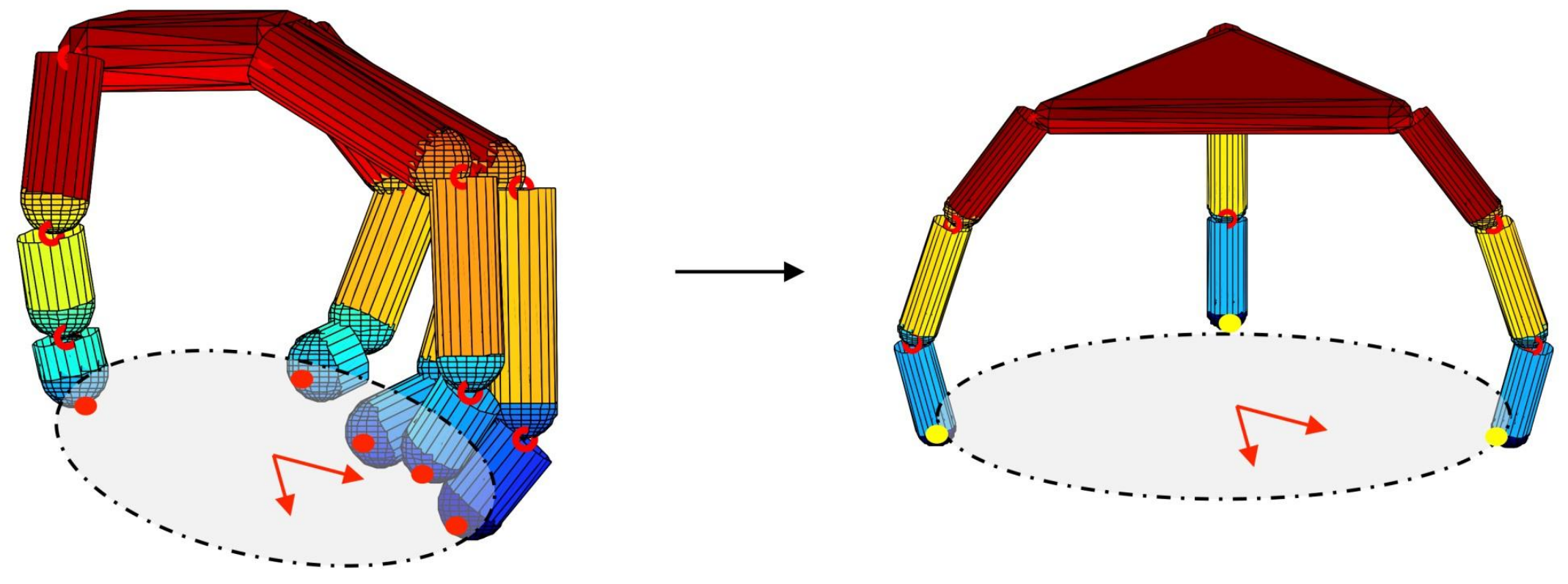

Figure 1: Mapping synergies from the human hand model (left) to the robotic hand (right). The reference points on the human hand model (red dots) allow the definition of the virtual object. Activating the human hand synergies, the object is moved and strained; its motion and strain can be evaluated from the velocities of the reference points. This motion and strain, scaled if necessary, are then imposed to the virtual object relative to the robotic hand, defined on the basis of the robotic reference points (yellow dots). The hand models in the figure are obtained using the SynGrasp Toolbox [99]. 
(a)

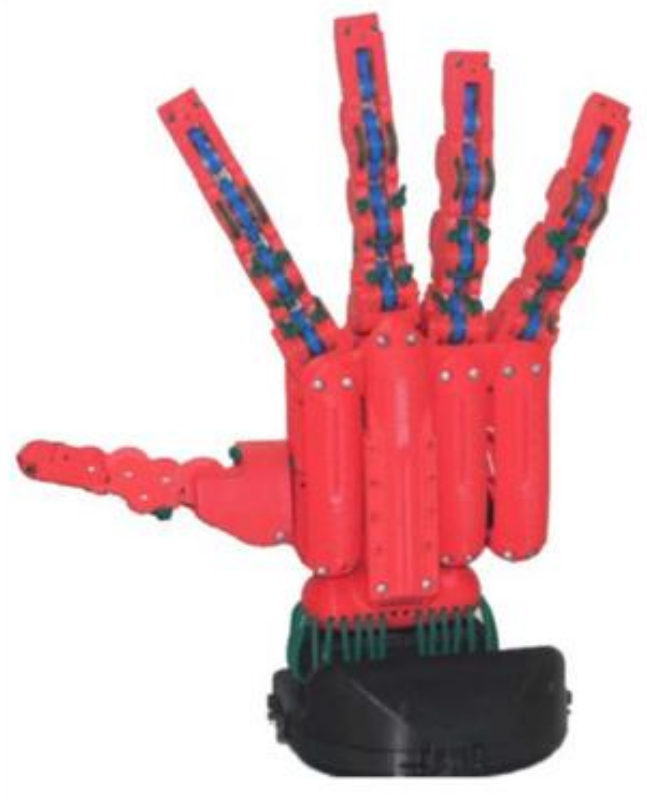

(c)
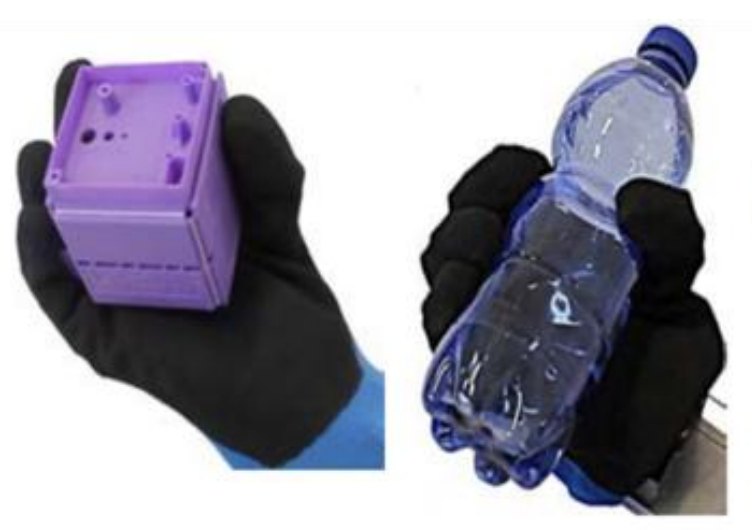

(b)

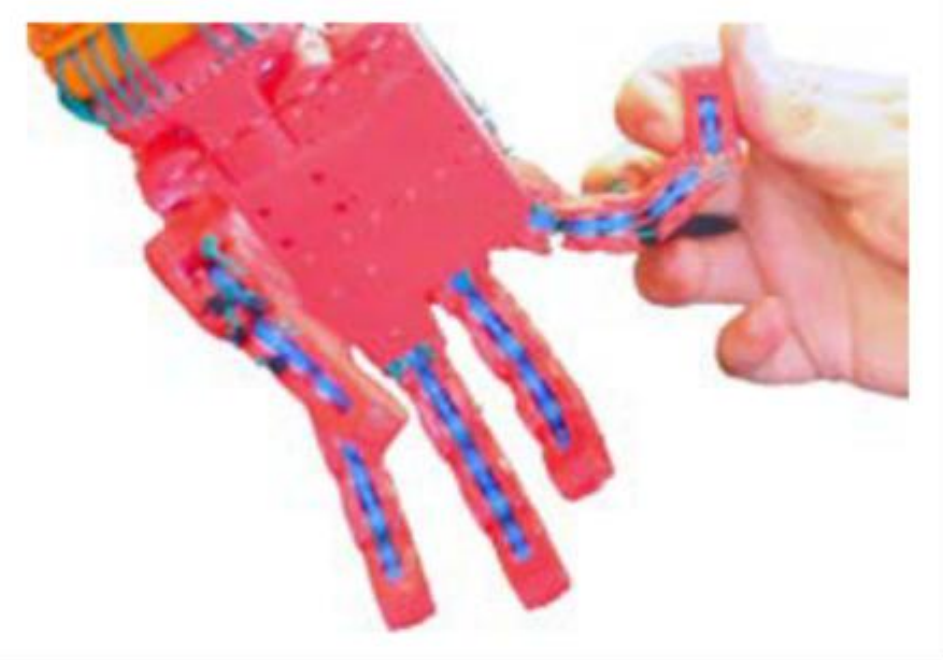

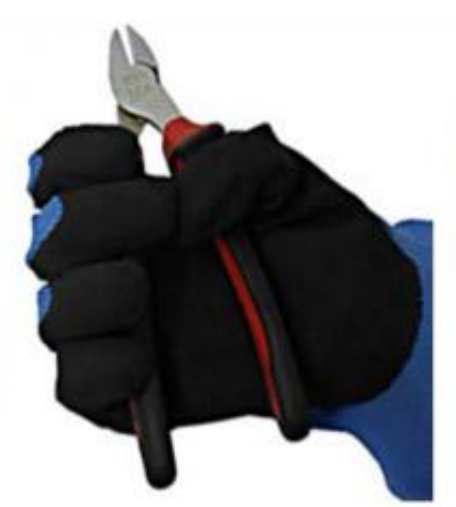

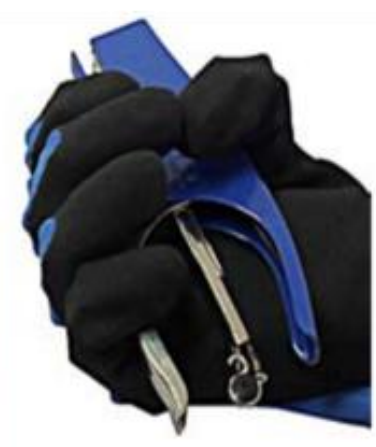

Figure 2: (a) Skeleton of the Pisa/IIT SoftHand advanced anthropomorphic hand prototype. (b) The SoftHand joints can withstand large forces in all directions, thus allowing the hand to automatically return to the initial configuration. (c) Examples of experimental grasps performed with the Pisa/IIT Softhand (Adapted from [109]) 